\title{
The Largest Laplacian Spectral Radius of Unicyclic Graphs with Fixed Diameter
}

\author{
Haixia Zhang \\ Department of Mathematics, Taiyuan University of Science and Technology, Taiyuan 030024, China \\ Correspondence should be addressed to Haixia Zhang; zhanghaixiass@hotmail.com
}

Received 27 September 2013; Accepted 11 November 2013

Academic Editor: Baolin Wang

Copyright (c) 2013 Haixia Zhang. This is an open access article distributed under the Creative Commons Attribution License, which permits unrestricted use, distribution, and reproduction in any medium, provided the original work is properly cited.

We identify graphs with the maximal Laplacian spectral radius among all unicyclic graphs with $n$ vertices and diameter $d$.

\section{Introduction}

Following [1], let $G=(V(G), E(G))$ be a simple undirected graph on $n$ vertices and $m$ edges (so $n=|V(G)|$ is its order and $m=|E(G)|$ is its size). For $v \in V(G), d_{G}(v)$ or $d(v)$ denotes the degree of $v$ and $N(v)$ denotes the set of all neighbors of vertex $v$. A pendant vertex is a vertex of degree 1 and a pendant edge is an edge incident with a pendant vertex. Let $P V(G)=$ $\left\{v: d_{G}(v)=1\right\}$. For two vertices $u$ and $v(u \neq v)$, the distance between $u$ and $v$ is the number of edges in the shortest path joining $u$ and $v$. The diameter of a graph is the maximum distance between any two vertices of $G$. Let $P=v_{0} v_{1}, \ldots, v_{s}$ $(s \geq 1)$ be a path of $G$ with $d\left(v_{1}\right)=\cdots=d\left(v_{s-1}\right)=2$ (unless $s=1)$. If $d\left(v_{0}\right), d\left(v_{s}\right) \geq 3$, then we call $P$ an internal path of $G$; if $d\left(v_{0}\right) \geq 3$ and $d\left(v_{s}\right)=1$, then we call $P$ a pendant path of $G$; if the subgraph induced by $V(P)$ in $G$ is $P$ itself, that is, $G[V(P)]=P$, then we call $P$ an induced path. Obviously, the shortest path between any two distinct vertices of $G$ is an induced path. We will use $G-v, G-u v$ to denote the graph obtained from $G$ by deleting a vertex $v \in V(G)$, or an edge $u v \in E(G)$, respectively (this notation is naturally extended if more than one vertex, or edge, is deleted).

Denote by $C_{n}$ and $P_{n}$ the cycle and the path with $n$ vertices, respectively. We call $G$ a unicyclic graph if $m=n$, where $n$ is the number of vertices and $m$ is the number of edges. We will use $\mathscr{C}_{n}^{d}$ to denote the sets of all unicyclic graphs with $n$ vertices and diameter $d$. Let $\diamond_{n}^{k}$ be a graph of order $n$ obtained from the cycle $C_{4}$ by attaching $n-d-2$ pendant edges and a path of length $d-k-1$ at one vertex of the cycle, and a path of length $k-1$ to another nonadjacent vertex of the cycle respectively, where $0 \leq k-1 \leq d-k-1$.

Let $L(G)=D(G)-A(G)$ be the Laplacian matrix, where $D(G)$ is the diagonal matrix and $A(G)$ is the adjacency matrix. The matrix $L(G)$ is real symmetric and positive semidefinite; the eigenvalues of $L(G)$ can be arranged as $\mu_{1}(G) \geq \cdots \geq$ $\mu_{n}(G)=0$, where the largest eigenvalue $\mu_{1}(G)$ is called the Laplacian spectral radius of $G$.

The investigation on the Laplacian spectral radius of graphs is an important topic in the theory of graph spectra. Recently, the problem concerning graphs with maximal Laplacian spectral radius of a given class of graphs has been studied extensively. $\mathrm{Li}$ et al. [2] determined those graphs which maximized Laplacian spectral radius among all bipartite graphs with (edge-) connectivity at most $k$ and characterized graphs of order $n$ with $k$ cut-edges, having Laplacian spectral radius equal to $n$. X. L. Zhang and H. P. Zhang [3] studied the largest Laplacian spectral radius of the bipartite graphs with $n$ vertices and $k$ cut edges and the bicyclic bipartite graphs, respectively. The Laplacian spectral radius of unicyclic graphs has been studied by many authors (see [4-6]). Liu et al. [7] determined the graphs with the largest Laplacian spectral radii among all unicyclic graphs and bicyclic graphs with $n$ vertices and $k$ pendant vertices. Hua et al. [8] determined extremal graphs with maximal Laplacian spectral radius among all unicyclic graphs with given order and given pendant vertices number.

In 2007, Liu et al. [9] determined graphs with the maximal spectral radius among all unicyclic graphs with $n$ vertices 
and diameter $d$. In 2012, He and Li [6] identified graphs with the maximal signless Laplacian spectral radius among all unicyclic graphs with $n$ vertices of diameter $d$. Next, Guo [4] considered the Laplacian spectral radius of unicyclic graphs with fixed diameter and proposed Conjecture 1.

In this paper, we prove the conjecture as Theorem 1.

Theorem 1. Let $G$ be a graph in $\mathscr{C}_{n}^{d}, 3 \leq d \leq n-2$. Consider the following.

(i) If $d$ is odd, $\mu_{1}(G) \leq \mu_{1}\left(\diamond_{n}^{\lfloor d / 2\rfloor}\right)$ and equality holds if and only if $G \cong \diamond_{n}^{\lfloor d / 2\rfloor}$.

(ii) If $d$ is even and $n-d-2=0,1, \mu_{1}(G) \leq \mu_{1}\left(\diamond_{n}^{\lfloor d / 2\rfloor}\right)$ and equality holds if and only if $G \cong \diamond_{n}^{\lfloor d / 2\rfloor}$.

(iii) If $d$ is even and $n-d-2 \geq 2, \mu_{1}(G) \leq \mu_{1}\left(\diamond_{n}^{\lfloor d / 2\rfloor-1}\right)$ and equality holds if and only if $G \cong \diamond_{n}^{\lfloor d / 2\rfloor-1}$.

The rest of this paper is organized as follows. In Section 2, we present some notations and lemmas which will be used later on. In Section 3, we determine graphs with the largest Laplacian spectral radius among all unicyclic graphs with $n$ vertices and diameter $d$.

\section{Lemmas}

In this section, we list some lemmas which will be used to prove our main results.

Lemma 2 (see [10]). Suppose that $u$, $v$ are two distinct vertices of a connected graph $G$. Let $G_{t}$ be the graph obtained from $G$ by attaching $t$ new paths $v v_{i 1} v_{i 2}, \ldots, v_{i q_{i}}(i=1,2, \ldots, t)$ at $v$. Let $X=\left(x_{1}, x_{2}, \ldots, x_{n}\right)^{T}$, where $x_{i}$ corresponds to the vertex $v_{i}(1 \leq i \leq n)$, be a unit eigenvector of $G_{t}$ corresponding to $\mu_{1}\left(G_{t}\right) \geq 4$. Let

$$
\begin{aligned}
G_{u}= & G_{t}-v v_{11}-v v_{21}-\cdots-v v_{t 1} \\
& +u v_{11}+u v_{21}+\cdots+u v_{t 1}
\end{aligned}
$$

If $\left|x_{u}\right| \geq\left|x_{v}\right|$, then $\mu_{1}\left(G_{u}\right) \geq \mu_{1}\left(G_{t}\right)$. Further, if $\left|x_{u}\right|>\left|x_{v}\right|$, then $\mu_{1}\left(G_{u}\right)>\mu_{1}\left(G_{t}\right)$.

Lemma 3 (see [10]). Let uv be a pendant edge of a connected graph $G$ with $n \geq 2$ vertices and let $v$ be a pendant vertex. Let $G_{1}, G_{2}, \ldots, G_{k}(k \geq 2)$ be $k$ disjoint connected graphs and let $v_{i}$ be a vertex of $G_{i}(i=1,2, \ldots, k)$. Let $G^{\prime}$ be the graph obtained by adding $k$ new edges $v v_{1}, v v_{2}, \ldots, v v_{k}$ among $G, G_{1}, G_{2}, \ldots, G_{k}$. Let

$$
\begin{aligned}
G^{*}= & G^{\prime}-v v_{1}-v v_{2}-\cdots-v v_{k} \\
& +u v_{1}+u v_{2}+\cdots+u v_{k} .
\end{aligned}
$$

(i) If $n=2$, then $\mu_{1}\left(G^{*}\right)=\mu_{1}\left(G^{\prime}\right)$.

(ii) If $n \geq 3$, then $\mu_{1}\left(G^{*}\right) \geq \mu_{1}\left(G^{\prime}\right)$, with equality if and only if either $\mu_{1}\left(G^{*}\right)=\mu_{1}(G)$ or there exists some $i$ ( $1 \leq$ $i \leq k)$ such that $\mu_{1}\left(G^{*}\right)=\mu_{1}\left(G_{i}\right)$.
Let $v$ be a vertex of a connected graph $G$ with at least two vertices. Let $G_{k, l}(l \geq k \geq 1)$ be the graph obtained from $G$ by attaching two new paths $P: v\left(=v_{0}\right) v_{1} v_{2}, \ldots, v_{k}$ and $Q$ : $v\left(=v_{0}\right) u_{1} u_{2}, \ldots, u_{l}$ of length $k$ and $l$, respectively, at $v$, where $u_{1}, u_{2}, \ldots, u_{l}$ and $v_{1}, v_{2}, \ldots, v_{k}$ are distinct new vertices. Let $G_{k-1, l+1}=G_{k, l}-v_{k-1} v_{k}+u_{l} v_{k}$.

Lemma 4 (see [11]). Let $G$ be a connected graph on $n \geq$ 2 vertices and $v$ be a vertex of $G$. Let $G_{k, l}$ be the graph defined as previously mentioned. If $l \geq k \geq 1$, then $\mu_{1}\left(G_{k-1, l+1}\right) \leq \mu_{1}\left(G_{k, l}\right)$, with equality if and only if there exists a unit eigenvector of $G_{k, l}$ corresponding to $\mu_{1}\left(G_{k, l}\right)$ taking the value 0 on vertex $v$.

Lemma 5 (see [1]). Let $G^{\prime}$ be a graph obtained by deleting an edge from the graph $G$. Then $\mu_{i}(G) \geq \mu_{i}\left(G^{\prime}\right) \geq \mu_{i+1}(G), i=$ $1, \ldots, n-1$.

Let $S_{3}^{i}$ be a graph obtained from the cycle $C_{3}$ by attaching $i$ pendant edges at one vertex of the cycle $C_{3}$.

Lemma 6 (see [5]). Let $G$ be a unicyclic graph on $n$ vertices; then $\mu_{1}(G) \geq \mu_{1}\left(C_{n}\right)$; when $n \neq 4$, the equality holds if and only if $G \cong C_{n}$; when $n=4$, the equality holds if and only if $G \cong C_{4}$, $G \cong S_{3}^{1}$.

Lemma 7. Let $G$ be a connected graph with at least one edge, let $\Delta(G)$ be its maximal degree, and let $d_{i}$ be the degree of vertex $v_{i}$ and $m_{i}=\sum_{v_{j} \in N\left(v_{i}\right)} d_{j} / d_{i}$; then

(i) $\mu_{1}(G) \geq \Delta(G)+1$; the equality holds if and only if $\Delta(G)=n-1[12]$;

(ii) $\mu_{1}(G) \leq \max \left\{d_{i}+m_{i} \mid v_{i} \in V(G)\right\}$; the equality holds if and only if $G$ is regular or semiregular bipartite graph [13].

Let $L_{v_{i}}(G)$ be the principal submatrix obtained from $L(G)$ by deleting the corresponding row and column of $v_{i}$. Generally, let $L_{S}(G)$ be the principal submatrix obtained from $L(G)$ by deleting the corresponding rows and columns of all vertices of $S$. For any square matrix $B$, denote by $\Phi(B)=$ $\Phi(B, x)=\operatorname{det}(x I-B)$ the characteristic polynomial of $B$. In particular, if $B=L(G)$, we write $\Phi(L(G))$ by $\Phi(G)$ for convenience. If $G=u$, then suppose that $\Phi\left(L_{u}(G)\right)=1$.

Let $G=G_{1} u: v G_{2}$ be the graph obtained by joining the vertex $u$ of the graph $G_{1}$ to the vertex of $v$ of the graph $G_{2}$ by an edge. We call $G$ a connected sum of $G_{1}$ at $u$ and $G_{2}$ at $v$.

Lemma 8. Let $G_{1}$ and $G_{2}$ be two graphs. If $\Phi\left(G_{1}\right)>\Phi\left(G_{2}\right)$ for $x \geq \mu_{1}\left(G_{2}\right)$, then $\mu_{1}\left(G_{1}\right)<\mu_{1}\left(G_{2}\right)$. (In general, let $f(x)$ and $g(x)$ be polynomials with positive leading coefficients. If $f(x)>g(x)$ for $x \geq \mu_{1}(g(x))$, then $\mu_{1}(f(x))<\mu_{1}(g(x))$, where $\mu_{1}(g(x))$ and $\mu_{1}(f(x))$ are the largest roots of $g(x)=0$ and $f(x)=0$, resp.)

Proof. If $\mu_{1}\left(G_{1}\right) \geq \mu_{1}\left(G_{2}\right)$, then $\left.\Phi\left(G_{1}\right)\right|_{x=\mu_{1}\left(G_{1}\right)}=0$, $\left.\Phi\left(G_{2}\right)\right|_{x=\mu_{1}\left(G_{1}\right)} \geq 0$, a contradiction. 
Lemma 9 (see [14]). Let $G=G_{1} u: v G_{2}$ be a connected sum of $G_{1}$ at $u$ and $G_{2}$ at $v$; then

$$
\begin{aligned}
\Phi(G)= & \Phi\left(G_{1}\right) \Phi\left(G_{2}\right)-\Phi\left(G_{1}\right) \Phi\left(L_{v}\left(G_{2}\right)\right) \\
& -\Phi\left(G_{2}\right) \Phi\left(L_{u}\left(G_{1}\right)\right) .
\end{aligned}
$$

Lemma 10 (see [14]). Let $G$ be a connected graph with $n$ vertices which consists of a subgraph $H$ and $n-|V(H)|$ distinct pendant edges (not in $H$ ) attaching to a vertex $v$ in $H$. Then

$$
\begin{aligned}
\Phi(G)= & (x-1)^{n-|V(H)|} \Phi(H) \\
& -(n-|V(H)|) x(x-1)^{n-|V(H)|-1} \Phi\left(L_{v}(H)\right) .
\end{aligned}
$$

Lemma 11 (see [15]). Let $D_{n}(n \geq 1)$ be the matrix obtained from $L\left(P_{n+2}\right)$ by deleting the rows and columns corresponding to two pendant vertices of $P_{n+2}$; suppose that $\Phi\left(D_{0}\right)=1$, $\Phi\left(D_{-n}\right)=0$; then

(i) $x \Phi\left(D_{n-1}\right)=\Phi\left(P_{n}\right)$;

(ii) $\Phi\left(D_{n+1}\right)=(x-2) \Phi\left(D_{n}\right)-\Phi\left(D_{n-1}\right)$;

(iii) $\Phi\left(D_{m+1}\right) \Phi\left(D_{n}\right)-\Phi\left(D_{m}\right) \Phi\left(D_{n+1}\right)=\Phi\left(D_{m}\right) \Phi\left(D_{n-1}\right)-$ $\Phi\left(D_{m-1}\right) \Phi\left(D_{n}\right),(n, m \geq 1)$

(iv) $\Phi\left(C_{n}\right)=\Phi\left(D_{n}\right)-\Phi\left(D_{n-2}\right)+2(-1)^{n+1}$.

From Lemma 11(i), all eigenvalues of $D_{n}$ are $2+$ $2 \cos (i \pi /(n+1))$, where $1 \leq i \leq n$. Other characterizations of $\Phi\left(D_{n}\right)$ can be shown below.

Lemma 12. Let $D_{n}(n \geq 1)$ be the matrix as above. Consider the following.

(i) If $n \geq 1$, then $\Phi\left(D_{n}\right)>\Phi\left(D_{n-1}\right)$ when $x \geq 4$.

(ii) If $n \geq 1$, then $\Phi\left(D_{n}\right)>2 \Phi\left(D_{n-1}\right)$ when $x \geq 5$.

(iii) $\Phi\left(D_{m}\right) \Phi\left(D_{n}\right)-\Phi\left(D_{m-1}\right) \Phi\left(D_{n+1}\right)=\Phi\left(D_{n-m}\right)$, where $0 \leq m \leq n$.

Proof. From Lemma 11(ii), it is easy to prove (i) and (ii) by introduction on $n$.

By Lemma 11(iii), we have

$$
\begin{aligned}
\Phi & \left(D_{m}\right) \Phi\left(D_{n}\right)-\Phi\left(D_{m-1}\right) \Phi\left(D_{n+1}\right) \\
& =\Phi\left(D_{m-1}\right) \Phi\left(D_{n-1}\right)-\Phi\left(D_{m-2}\right) \Phi\left(D_{n}\right) \\
& =\Phi\left(D_{m-2}\right) \Phi\left(D_{n-2}\right)-\Phi\left(D_{m-3}\right) \Phi\left(D_{n-1}\right) \\
& =\cdots=\Phi\left(D_{0}\right) \Phi\left(D_{n-m}\right)-\Phi\left(D_{-1}\right) \Phi\left(D_{n-m+1}\right) \\
& =\Phi\left(D_{n-m}\right)
\end{aligned}
$$

as desired.

Lemma 13. Suppose that $u, v$ are two adjacent vertices of the cycle $C_{q}$, where $q$ is even. Let $H_{k, l}(l \geq k \geq 1)$ be the graph obtained from $C_{q}$ by attaching two new paths $P: v(=$ $\left.v_{0}\right) v_{1} v_{2}, \ldots, v_{k}$ and $Q: u\left(=u_{0}\right) u_{1} u_{2}, \ldots, u_{l}$ of length $k$ and $l$ at $v$ and $u$, respectively, where $u_{1}, u_{2}, \ldots, u_{l}$ and $v_{1}, v_{2}, \ldots, v_{k}$ are distinct new vertices. Let $H_{k-1, l+1}=H_{k, l}-v_{k-1} v_{k}+u_{l} v_{k}$. Then $\mu_{1}\left(H_{k-1, l+1}\right)<\mu_{1}\left(H_{k, l}\right)$.
Proof. Using Lemma 9, we have

$$
\begin{aligned}
& \Phi(\left.H_{k-1, l+1}\right)-\Phi\left(H_{k, l}\right) \\
&=x\left.\Phi\left(L_{v_{k-1}}\left(H_{k-1, l}\right)\right)-\Phi\left(L_{u_{l}}\left(H_{k-1, l}\right)\right)\right] \\
&=x\left\{\left[(x-1) \Phi\left(L_{v_{k-1}, u_{l}}\left(H_{k-1, l}\right)\right)-\Phi\left(L_{v_{k-1}, u_{l}, u_{l-1}}\left(H_{k-1, l}\right)\right)\right]\right. \\
& \quad-\left[(x-1) \Phi\left(L_{u_{l}, v_{k-1}}\left(H_{k-1, l}\right)\right)\right. \\
&\left.\left.\quad-\Phi\left(L_{u_{l}, v_{k-1}, v_{k-2}}\left(H_{k-1, l}\right)\right)\right]\right\} \\
&=\cdots=x\left[b_{k, l}(x)-a_{k, l}(x)\right],
\end{aligned}
$$

where

$$
\begin{aligned}
a_{k, l}(x)= & {\left[\Phi\left(C_{q}\right)-2 \Phi\left(D_{q^{-1}}\right)+\Phi\left(D_{q-2}\right)\right] \Phi\left(D_{l-k}\right) } \\
& -\left[\Phi\left(D_{q-1}\right)-\Phi\left(D_{q-2}\right)\right] \Phi\left(D_{l-k-1}\right), \\
b_{k, l}(x)= & {\left[\Phi\left(D_{q-1}\right)-\Phi\left(D_{q-2}\right)\right] \Phi\left(D_{l-k+1}\right) } \\
& -\Phi\left(D_{q-2}\right) \Phi\left(D_{l-k}\right) .
\end{aligned}
$$

From Lemmas 11(ii) and 11(iv), (6) becomes

$$
\begin{aligned}
& \Phi\left(H_{k-1, l+1}\right)-\Phi\left(H_{k, l}\right) \\
& \quad=x \Phi\left(D_{l-k}\right)\left[(x-2) \Phi\left(D_{q-2}\right)-2 \Phi\left(D_{q-3}\right)+2\right],
\end{aligned}
$$

which is greater than 0 when $x \geq 4$ by Lemma 12(i). And $\mu_{1}\left(H_{k, l}\right)>4$ follows from Lemma 7(i). Thus $\mu_{1}\left(H_{k-1, l+1}\right)<$ $\mu_{1}\left(H_{k, l}\right)$ holds by Lemma 8.

For $G \in \mathscr{C}_{n}^{d}$, we have $n \geq 3$ and $1 \leq d \leq n-2$. If $d=1$, then $G \cong C_{3}$. If $d=2$, then $G \cong C_{4}, G \cong C_{5}$ or $G \cong S_{3}^{n-3}$. By Lemma $7, \mu_{1}\left(S_{3}^{n-3}\right)$ has the largest Laplacian spectral radius.

Therefore, in the following, we assume that $3 \leq d \leq n-2$.

Let $H_{0}$ be the unicyclic graph of order $d+2$ shown in Figure 1. Let $H_{0}\left(p_{2}, \ldots, p_{d}, p_{d+2}\right)$ be a graph of order $n$ obtained from $H_{0}$ by attaching $p_{i}$ pendant vertices to each $v_{i} \in V\left(H_{0}\right) \backslash\left\{v_{1}, v_{d+1}\right\}$, respectively, where $p_{d+2}=0$ when $k=1$ or $k=d$. Denote that

$$
\begin{gathered}
\widetilde{\mathscr{H}}_{n}^{d}=\left\{H_{0}\left(p_{2}, \ldots, p_{d}, p_{d+2}\right): \sum_{i=2}^{d} p_{i}+p_{d+2}=n-d-2\right\}, \\
\overline{\mathscr{H}}_{n}^{d}=\left\{H_{0}\left(0, \ldots, 0, p_{i}, 0, \ldots, 0\right)=H_{0}\left(p_{i}\right): p_{i}=n-d-2\right\} .
\end{gathered}
$$

Lemma 14. Let $G \in \widetilde{\mathscr{H}}_{n}^{d}$. Then there is a graph $G^{*} \in \overline{\mathscr{H}}_{n}^{d}$ such that $\mu_{1}\left(G^{*}\right) \geq \mu_{1}(G)$.

Proof. Let $G \in \widetilde{\mathscr{H}}_{n}^{d} \backslash \overline{\mathscr{H}}_{n}^{d}$ and let $X=\left(x_{1}, x_{2}, \ldots, x_{n}\right)^{T}$ be a unit eigenvector of $\mu_{1}(G)$, where $x_{i}$ corresponds to the vertex $v_{i}(1 \leq i \leq n)$. Let $t=\left|\left\{p_{i}: p_{i} \neq 0\right\}\right|$. Then $t \geq 2$. Let $p_{i}, p_{j} \neq 0, i<j$. Assume, without loss of generality, that $\left|x_{i}\right| \geq\left|x_{j}\right|$. Let $N\left(v_{j}\right) \cap P V(G)=\left\{u_{1}, u_{2}, \ldots, u_{p_{j}}\right\}$. Let

$$
G_{1}^{*}=G-v_{j} u_{1}-\cdots-v_{j} u_{p_{j}}+v_{i} u_{1}+\cdots+v_{i} u_{p_{j}} .
$$




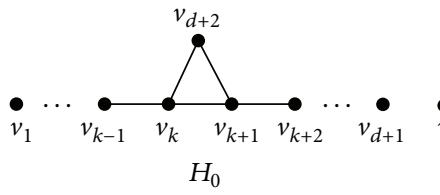

(a)

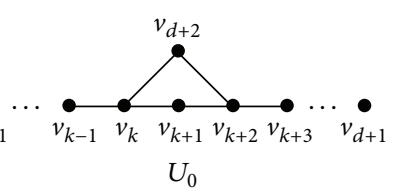

(b)
Figure 1

By Lemma 2, we have $\mu_{1}\left(G^{*}\right) \geq \mu_{1}(G)$. Note that $G_{1} \in \overline{\mathscr{H}}_{n}^{d}$ for $t=2$ and $G_{1} \in \widetilde{\mathscr{H}}_{n}^{d} \backslash \overline{\mathscr{H}}_{n}^{d}$ for $t>2$. If $t>2$, then we will use $G_{1}^{*}$ to repeat the above step until the cardinality of $p_{i}$, being nonzero, is only one. So we have $G_{2}^{*}, G_{3}^{*}, \ldots, G_{t-1}^{*}$ and $\mu_{1}\left(G_{2}^{*}\right) \leq \mu_{1}\left(G_{3}^{*}\right) \leq \cdots \leq \mu_{1}\left(G_{t-1}^{*}\right)$. Note that $G_{t-1}^{*} \in \overline{\mathscr{H}}_{n}^{d}$, and hence the lemma holds.

Lemma 15. For any $G \in \overline{\mathscr{H}}_{n}^{d}, \mu_{1}(G) \leq \mu_{1}\left(H_{0}\left(p_{k+1}\right)\right)$, where $0 \leq k-1 \leq d-k$; the equality holds if and only if $G \cong H_{0}\left(p_{k+1}\right)$.

Proof. Suppose that $n-d-2=t$. If $t=0$, the result is obvious.

If $t \geq 1$, by Lemma 7 , we have

$$
\begin{aligned}
\mu_{1}(G)< & \max \left\{\max \left\{d_{i}+m_{i} \mid v_{i} \in V(G)\right\} \mid G \in \overline{\mathscr{H}}_{n}^{d},\right. \\
& i \notin\{d+2, k, k+1\}\} \\
& =t+2+\frac{2+3+t}{t+2} \leq t+4=\Delta\left(H_{0}\left(p_{k+1}\right)\right)+1 \\
< & \mu_{1}\left(H_{0}\left(p_{k+1}\right)\right) .
\end{aligned}
$$

Case 1. $k-1<d-k$.

When $i=d+2$, let $N\left(v_{i}\right) \cap P V(G)=\left\{u_{1}, u_{2}, \ldots, u_{t}\right\}$. Let

$$
G^{*}=\left\{\begin{array}{lr}
H_{0}\left(p_{i}\right)-v_{i} u_{1}-\cdots-v_{i} u_{t}+v_{k} u_{1}+\cdots+v_{k} u_{t}, \\
H_{0}\left(p_{i}\right)-v_{k-1} v_{k}+v_{k-1} v_{i}, & \left|x_{k}\right| \geq\left|x_{i}\right|, \\
& \left|x_{k}\right|<\left|x_{i}\right| .
\end{array}\right.
$$

Then, in all cases, $G^{*} \cong H_{0}\left(p_{k}\right)$. Thus by Lemma 2 , $\mu_{1}\left(H_{0}\left(p_{d+2}\right)\right) \leq \mu_{1}\left(H_{0}\left(p_{k}\right)\right)$.

Next, we show that $\Phi\left(H_{0}\left(p_{k}\right)\right)>\Phi\left(H_{0}\left(p_{k+1}\right)\right)$ for $x \geq$ $\mu_{1}\left(H_{0}\left(p_{k+1}\right)\right)$. Because $H_{0}-v_{k} \cong P_{k-1} \bigcup P_{d-k+2}$, we can get $\Phi\left(H_{0}\right)$ in which the rows and columns correspond to vertices as the ordering $v_{1}, \ldots, v_{k-1}, v_{d+2}, v_{k+1}, v_{k+2}, \ldots, v_{d+1}$. Furthermore, let $E_{11}=\left[e_{i, j}\right]$ be a square matrix of order $k-1$, where $e_{k-1, k-1}=1$ and $e_{i, j}=0$ whenever $i \neq k-1$ and $j \neq k-1$; let $F_{k k}=\left[f_{i, j}\right]$ be a square matrix of order $d-k+2$, where $f_{k, k}=1$ and $f_{i, j}=0$ whenever $i \neq k$ and $j \neq k$. Then

$$
L_{v_{k}}\left(H_{0}\right)=\left(\begin{array}{cc}
L\left(P_{k-1}\right)+E_{11} & \mathbf{0} \\
\mathbf{0} & L\left(P_{d-k+2}\right)+F_{11}+F_{22}
\end{array}\right) .
$$

Hence,

$$
\begin{aligned}
\Phi & \left(L_{v_{k}}\left(H_{0}\right)\right) \\
& =\Phi\left(L\left(P_{k-1}\right)+E_{11}\right) \Phi\left(L\left(P_{d-k+2}\right)+F_{11}+F_{22}\right) .
\end{aligned}
$$

In order to simplify the notation, we denote $\Phi\left(L\left(P_{k-1}\right)+E_{11}\right)$ and $\Phi\left(L\left(P_{d-k+2}\right)+F_{11}+F_{22}\right)$ by $f_{k-1,1}(x)$ and $f_{d-k+2,2}(x)$, respectively. Similarly,

$$
\begin{aligned}
\Phi\left(L_{v_{k+1}}\left(H_{0}\right)\right) \\
\quad=\Phi\left(L\left(P_{d-k}\right)+E_{11}\right) \Phi\left(L\left(P_{k+1}\right)+F_{11}+F_{22}\right) \\
\quad=f_{d-k, 1}(x) f_{k+1,2}(x) .
\end{aligned}
$$

In general, by Lemma 11(ii), we have

$$
\begin{gathered}
f_{n_{1}, 1}(x)=\Phi\left(D_{n_{1}}\right)+\Phi\left(D_{n_{1}-1}\right), \\
f_{n_{2}, 2}(x)=(x-1)(x-3) \Phi\left(D_{n_{2}-2}\right)-(2 x-3) \Phi\left(D_{n_{2}-3}\right) .
\end{gathered}
$$

Hence, by Lemma 12(iii),

$$
\begin{aligned}
\Phi & \left(L_{v_{k+1}}\left(H_{0}\right)\right)-\Phi\left(L_{v_{k}}\left(H_{0}\right)\right) \\
= & f_{d-k, 1}(x) f_{k+1,2}(x)-f_{k-1,1}(x) f_{d-k+2,2}(x) \\
= & (x-1)(x-3) \\
& \quad \times\left[\Phi\left(D_{k-1}\right) \Phi\left(D_{d-k-1}\right)-\Phi\left(D_{k-2}\right) \Phi\left(D_{d-k}\right)\right] \\
& +(2 x-3)\left[\Phi\left(D_{k-1}\right) \Phi\left(D_{d-k-1}\right)-\Phi\left(D_{k-2}\right) \Phi\left(D_{d-k}\right)\right] \\
= & x(x-2) \Phi\left(D_{d-2 k}\right) .
\end{aligned}
$$

From (17) and Lemma 10,

$$
\begin{aligned}
& \Phi\left(H_{0}\left(p_{k}\right)\right)-\Phi\left(H_{0}\left(p_{k+1}\right)\right) \\
& \quad=t(x-2)(x-1)^{t-1} x^{2} \Phi\left(D_{d-2 k}\right)>0
\end{aligned}
$$

holds for $x \geq \mu_{1}\left(H_{0}\left(p_{k+1}\right)\right)$.

Thus $\mu_{1}\left(H_{0}\left(p_{k}\right)\right)<\mu_{1}\left(H_{0}\left(p_{k+1}\right)\right)$ follows from Lemma 8 .

Case 2. $k-1=d-k$.

First note that $H_{0}\left(p_{k}\right) \cong H_{0}\left(p_{k+1}\right)$.

Next, since $H_{0}-v_{d+2}=P_{d+1}$, we can derive $\Phi\left(L_{v_{d+2}}\left(H_{0}\right)\right)$ in which the rows and columns correspond to vertices as the ordering $v_{1}, \ldots, v_{k-1}, v_{k}, v_{k+1}, \ldots, v_{d+1}$. Then

$$
\begin{aligned}
\Phi( & \left.L_{v_{d+2}}\left(H_{0}\right)\right) \\
= & {\left[\Phi\left(D_{k-1}\right)+\Phi\left(D_{k-2}\right)\right] } \\
& \times\left[\left(x^{2}-5 x+5\right) \Phi\left(D_{k-1}\right)-(2 x-5) \Phi\left(D_{k-1}\right)\right] \\
& -\left[\Phi\left(D_{k-2}\right)+\Phi\left(D_{k-3}\right)\right] \\
& \times\left[(x-2) \Phi\left(D_{k-1}\right)-2 \Phi\left(D_{k-2}\right)\right] .
\end{aligned}
$$


Combining Lemma 10 with (15) and (19), we get

$$
\begin{aligned}
& \Phi\left(H_{0}\left(p_{d+2}\right)\right)-\Phi\left(H_{0}\left(p_{k+1}\right)\right) \\
& =t(x-1)^{t-1} x \Phi\left(D_{k-2}\right)\left[(x-2) \Phi\left(D_{k-1}\right)-2 \Phi\left(D_{k-2}\right)\right],
\end{aligned}
$$

which is greater than 0 when $x \geq \mu_{1}\left(H_{0}\left(p_{k+1}\right)\right)$ by Lemma 12(i). Thus, by Lemma $8, \mu_{1}\left(H_{0}\left(p_{d+2}\right)\right)$ $\mu_{1}\left(H_{0}\left(p_{k+1}\right)\right)$ holds. Hence, the proof is completed.

Let $\Delta_{n}^{k}$ be a graph of order $n$ obtained from a triangle by attaching $n-d-2$ pendant edges and a path of length $d-k$ at one vertex of the triangle, and a path of length $k-1$ to another vertex of the triangle, respectively, where $0 \leq k-1 \leq d-k$.

Lemma 16. $\mu_{1}\left(\Delta_{n}^{k-1}\right)<\mu_{1}\left(\Delta_{n}^{k}\right)$, where $2 \leq k \leq\lceil d / 2\rceil$.

Proof. Suppose that $n-d-2=t$; by Lemma 7(i), $\mu_{1}\left(\Delta_{n}^{k}\right)>$ $t+4$. Let $H^{*}=H_{0}-v_{1}$; by Lemma 9,

$$
\begin{gathered}
\Phi\left(\Delta_{d+2}^{k-1}\right)-\Phi\left(\Delta_{d+2}^{k}\right) \\
=x\left[\Phi\left(L_{v_{2}}\left(H^{*}\right)\right)-\Phi\left(L_{v_{d+1}}\left(H^{*}\right)\right)\right] \\
=x\left[(x-1) \Phi\left(L_{v_{2}, v_{d+1}}\left(H^{*}\right)\right)-\Phi\left(L_{v_{2}, v_{d+1}, v_{d}}\left(H^{*}\right)\right)\right. \\
\left.\quad-(x-1) \Phi\left(L_{v_{d+1}, v_{2}}\left(H^{*}\right)\right)+\Phi\left(L_{v_{d+1}, v_{2}, v_{3}}\left(H^{*}\right)\right)\right] \\
=\cdots=x\left[g_{d, k}(x)-f_{d, k}(x)\right],
\end{gathered}
$$

where

$$
\begin{aligned}
f_{d, k}(x)= & \left(x^{3}-8 x^{2}+18 x-8\right) \Phi\left(D_{d-2 k+1}\right) \\
& -\left(x^{2}-5 x+5\right) \Phi\left(D_{d-2 k}\right), \\
g_{d, k}(x)= & \left(x^{2}-5 x+5\right) \Phi\left(D_{d-2 k+2}\right) \\
& -(x-2) \Phi\left(D_{d-2 k+1}\right) .
\end{aligned}
$$

By Lemmas 10 and 11(ii) and (15) and (21),

$$
\begin{aligned}
\Phi & \left(\Delta_{n}^{k-1}\right)-\Phi\left(\Delta_{n}^{k}\right) \\
= & (x-1)^{t}\left[\Phi\left(\Delta_{d+2}^{k-1}\right)-\Phi\left(\Delta_{d+2}^{k}\right)\right] \\
& -t x(x-1)^{t-1}\left[\Phi\left(L_{v_{k}}\left(\Delta_{d+2}^{k-1}\right)\right)-\Phi\left(L_{v_{k+1}}\left(\Delta_{d+2}^{k}\right)\right)\right] \\
= & (x-1)^{t} x^{2}(x-4) \Phi\left(D_{d-2 k+1}\right)-t x(x-1)^{t-1} \\
& \times\left[f_{d-k+1,1}(x) f_{k, 2}(x)-f_{d-k, 1}(x) f_{k+1,2}(x)\right] \\
= & x^{2}(x-1)^{t-1} \\
& \times\left[(x-1)(x-t-4) \Phi\left(D_{d-2 k+1}\right)+t(x-2) \Phi\left(D_{d-2 k}\right)\right] \\
> & 0
\end{aligned}
$$

for $x \geq \mu_{1}\left(\Delta_{n}^{k}\right)$.

So $\mu_{1}\left(\Delta_{n}^{k-1}\right)<\mu_{1}\left(\Delta_{n}^{k}\right)$ follows from Lemma 8 .

In view of Lemma 16, the next corollary is obvious.
Corollary 17. $\mu_{1}\left(\Delta_{n}^{k}\right) \leq \mu_{1}\left(\Delta_{n}^{\lceil d / 2\rceil}\right)$, where $1 \leq k \leq\lceil d / 2\rceil$; the equality holds if and only if $\Delta_{n}^{k} \cong \Delta_{n}^{\lceil d / 2\rceil}$.

Let $U_{0}$ be the unicyclic graph of order $d+2$ shown in Figure 1. Let $U_{0}\left(p_{2}, \ldots, p_{d}, p_{d+2}\right)$ be a graph of order $n$ obtained from $U_{0}$ by attaching $p_{i}$ pendant vertices to each $v_{i} \in V\left(U_{0}\right) \backslash\left\{v_{1}, v_{d+1}\right\}$, respectively. Denote

$$
\begin{gathered}
\widetilde{\mathscr{U}}_{n}^{d}=\left\{U_{0}\left(p_{2}, \ldots, p_{d}, p_{d+2}\right): \sum_{i=2}^{d} p_{i}+p_{d+2}=n-d-2\right\}, \\
\overline{\mathcal{U}}_{n}^{d}=\left\{U_{0}\left(0, \ldots, 0, p_{i}, 0, \ldots, 0\right)=U_{0}\left(p_{i}\right): p_{i}=n-d-2\right\} .
\end{gathered}
$$

Lemma 18. Let $G \in \widetilde{\mathscr{U}}_{n}^{d}$. Then there is a graph $G^{*} \in \overline{\mathscr{U}}_{n}^{d}$ such that $\mu_{1}\left(G^{*}\right) \geq \mu_{1}(G)$.

Proof. The proof is similar to that of Lemma 14.

Lemma 19. For any $G \in \overline{\mathcal{U}}_{n}^{d}, \mu_{1}(G) \leq \mu_{1}\left(U_{0}\left(p_{k+2}\right)\right)$, where $0 \leq k-1 \leq d-k-1$; the equality holds if and only if $G \cong$ $U_{0}\left(p_{k+2}\right)$.

Proof. Suppose that $n-d-2=t$. If $t=0$, the result is trivial. If $t \geq 1$, by Lemma 7, we have

$\mu_{1}(G)<\max \left\{\max \left\{d_{i}+m_{i} \mid v_{i} \in V(G)\right\} \mid G \in \overline{\mathscr{U}}_{n}^{d}\right.$,

$$
\begin{gathered}
i \notin\{d+2, k, k+1, k+2\}\} \\
=t+2+\frac{2+3+t}{t+2} \leq t+4=\Delta\left(U_{0}\left(p_{k+2}\right)\right)+1 \\
<\mu_{1}\left(U_{0}\left(p_{k+2}\right)\right) .
\end{gathered}
$$

Case 1. $i=k$.

If $k-1=d-k-1, U_{0}\left(p_{k}\right) \cong U_{0}\left(p_{k+2}\right)$.

If $k-1<d-k-1$, we can obtain $\Phi\left(L_{v_{k+2}}\left(U_{0}\right)\right)$ in which the rows and columns correspond to vertices as the ordering $v_{d+2}, v_{k+1}, v_{k}, \ldots, v_{1}, v_{k+3}, \ldots, v_{d+1}$. Then by Lemma 11(ii),

$$
\begin{aligned}
& \Phi\left(L_{v_{k+2}}\left(U_{0}\right)\right) \\
& =(x-2)\left[\left(x^{2}-4 x+2\right) \Phi\left(D_{k-1}\right)-2(x-1) \Phi\left(D_{k-2}\right)\right] \\
& \quad \times\left[\Phi\left(D_{d-k-1}\right)+\Phi\left(D_{d-k-2}\right)\right] .
\end{aligned}
$$

Similarly,

$$
\begin{aligned}
& \Phi\left(L_{v_{k}}\left(U_{0}\right)\right) \\
& =(x-2)\left[\left(x^{2}-4 x+2\right) \Phi\left(D_{d-k-1}\right)-2(x-1) \Phi\left(D_{d-k-2}\right)\right] \\
& \quad \times\left[\Phi\left(D_{k-1}\right)+\Phi\left(D_{k-2}\right)\right] .
\end{aligned}
$$


Combining the two equations above with Lemmas 10 and 12(iii), we get

$$
\begin{aligned}
& \Phi\left(U_{0}\left(p_{k}\right)\right)-\Phi\left(U_{0}\left(p_{k+2}\right)\right) \\
& =t x(x-1)^{t-1}\left[\Phi\left(L_{v_{k+2}}\left(U_{0}\right)\right)-\Phi\left(L_{v_{k}}\left(U_{0}\right)\right)\right] \\
& =t x^{2}(x-2)^{2}(x-1)^{t-1} \Phi\left(D_{d-2 k-1}\right)>0
\end{aligned}
$$

for $x \geq \mu_{1}\left(U_{0}\left(p_{k+2}\right)\right)$.

From Lemma $8, \mu_{1}\left(U_{0}\left(p_{k}\right)\right)<\mu_{1}\left(U_{0}\left(p_{k+2}\right)\right)$ holds.

Case 2. $i=k+1, d+2$.

From Lemma 7, when $t \geq 2$, we have

$$
\begin{aligned}
& \mu_{1}\left(U_{0}\left(p_{k+1}\right)\right) \\
& \quad<\max \left\{d_{i}+m_{i} \mid v_{i} \in V\left(U_{0}\left(p_{k+1}\right)\right)\right\} \\
& \quad=t+2+\frac{3+3+t}{t+2} \leq t+4=\Delta\left(U_{0}\left(p_{k+2}\right)\right)+1 \\
& \quad<\mu_{1}\left(U_{0}\left(p_{k+2}\right)\right) .
\end{aligned}
$$

For $t=1$, we can obtain $\Phi\left(L_{v_{k+1}}\left(U_{0}\right)\right)$ in which the rows and columns correspond to vertices as the ordering $v_{1}, \ldots, v_{k-1}, v_{k}, v_{d+2}, v_{k+2}, \ldots, v_{d+1}$. Then

$$
\begin{aligned}
\Phi\left(L_{v_{k+1}}\left(U_{0}\right)\right)= & {\left[\Phi\left(D_{k-1}\right)+\Phi\left(D_{k-2}\right)\right] f(x) } \\
& -\left[\Phi\left(D_{k-2}\right)+\Phi\left(D_{k-3}\right)\right] g(x) \\
= & {[(x-2) g(x)-h(x)] \Phi\left(D_{k-1}\right) } \\
& -[2 g(x)+h(x)] \Phi\left(D_{k-2}\right),
\end{aligned}
$$

where

$$
\begin{gathered}
f(x)=\left(x^{3}-7 x^{2}+14 x-7\right) \Phi\left(D_{d-k-1}\right) \\
-\left(2 x^{2}-9 x+7\right) \Phi\left(D_{d-k-2}\right), \\
g(x)=(x-1)(x-3) \Phi\left(D_{d-k-1}\right)-(2 x-3) \Phi\left(D_{d-k-2}\right), \\
h(x)=(x-3) g(x)-f(x) \\
=(x-2) \Phi\left(D_{d-k-1}\right)-2 \Phi\left(D_{d-k-2}\right) .
\end{gathered}
$$

By (26) and (30),

$$
\begin{aligned}
& \Phi\left(L_{v_{k+2}}\left(U_{0}\right)\right)-\Phi\left(L_{v_{k+1}}\left(U_{0}\right)\right) \\
& =x\left\{(x-1) \Phi\left(D_{d-k-2}\right)\left[(x-3) \Phi\left(D_{k-1}\right)-2 \Phi\left(D_{k-2}\right)\right]\right. \\
& \left.\quad-\Phi\left(D_{d-k-1}\right) \Phi\left(D_{k-2}\right)\right\} .
\end{aligned}
$$

From Lemmas 11(ii) and 12(i), when $x \geq 5$,

$$
\begin{aligned}
& (x-1) \Phi\left(D_{d-k-2}\right)-\Phi\left(D_{d-k-1}\right) \\
& =\Phi\left(D_{d-k-2}\right)+\Phi\left(D_{d-k-3}\right)>0, \\
& (x-3) \Phi\left(D_{k-1}\right)-3 \Phi\left(D_{k-2}\right) \\
& = \begin{cases}\left(x^{2}-5 x+3\right) \Phi\left(D_{k-2}\right)-(x-3) \Phi\left(D_{k-3}\right)>0 & \text { if } k \geq 2, \\
x-3>0 & \text { if } k=1\end{cases}
\end{aligned}
$$

hold (since $\left(x^{2}-5 x+3\right)-(x-3)=x^{2}-6 x+6>0$ for $\left.x \geq 5\right)$.

By Lemma 9 and (32), when $x \geq 5$,

$$
\begin{aligned}
\Phi & \left(U_{0}\left(p_{k+1}\right)\right)-\Phi\left(U_{0}\left(p_{k+2}\right)\right) \\
& =x\left[\Phi\left(L_{v_{k+2}}\left(U_{0}\right)\right)-\Phi\left(L_{v_{k+1}}\left(U_{0}\right)\right)\right]>0 .
\end{aligned}
$$

From Lemma $8, \mu_{1}\left(U_{0}\left(p_{k+1}\right)\right)<\mu_{1}\left(U_{0}\left(p_{k+2}\right)\right)$ holds.

Hence, we complete the proof.

Let $\diamond_{n}^{k}$ be a graph of order $n$ obtained from the cycle $C_{4}$ by attaching $n-d-2$ pendant edges and a path of length $d-k-1$ at one vertex of the cycle and a path of length $k-1$ to another nonadjacent vertex of the cycle, respectively, where $0 \leq k-1 \leq d-k-1$.

Lemma 20. Let $\diamond_{n}^{k}$ be a graph defined as above; then

(i) if $k-1<d-k-1$, then $\mu_{1}\left(\diamond_{n}^{k-1}\right)<\mu_{1}\left(\diamond_{n}^{k}\right)$, where $2 \leq k \leq\lfloor d / 2\rfloor$

(ii) if $k-1=d-k-1($ i.e., $k=\lfloor d / 2\rfloor)$, then

(a) when $n-d-2=0$, or $1, \mu_{1}\left(\diamond_{n}^{k-1}\right)<\mu_{1}\left(\diamond_{n}^{k}\right)$;

(b) when $n-d-2 \geq 2, \mu_{1}\left(\diamond_{n}^{k}\right)<\mu_{1}\left(\diamond_{n}^{k-1}\right)$.

Proof. Suppose that $n-d-2=t$; by Lemma $7(\mathrm{i}), \mu_{1}\left(\diamond_{n}^{k}\right)>t+4$ holds. Let $U^{*}=U_{0}-v_{1}$; by Lemma 9,

$$
\begin{aligned}
\Phi & \left(\diamond_{d+2}^{k-1}\right)-\Phi\left(\diamond_{d+2}^{k}\right) \\
& =x\left[\Phi\left(L_{v_{2}}\left(U^{*}\right)\right)-\Phi\left(L_{v_{d+1}}\left(U^{*}\right)\right)\right] \\
& =x\left[\Phi\left(L_{v_{d+1}, v_{2}, v_{3}}\left(U^{*}\right)\right)-\Phi\left(L_{v_{2}, v_{d+1}, v_{d}}\left(U^{*}\right)\right)\right] \\
& =\cdots=x\left[s_{d, k}(x)-t_{d, k}(x)\right]
\end{aligned}
$$

where

$$
\begin{aligned}
s_{d, k}(x)=(x-2)[ & (x-1)(x-4) \Phi\left(D_{d-2 k+1}\right) \\
& \left.-(x-2) \Phi\left(D_{d-2 k}\right)\right], \\
t_{d, k}(x)=(x-2)[ & (x-3)\left(x^{2}-5 x+2\right) \Phi\left(D_{d-2 k}\right) \\
& \left.-(x-1)(x-4) \Phi\left(D_{d-2 k-1}\right)\right] .
\end{aligned}
$$


By Lemmas 10 and 11(ii) and (26) and (35),

$$
\begin{aligned}
\Phi & \left(\diamond_{n}^{k-1}\right)-\Phi\left(\diamond_{n}^{k}\right) \\
= & (x-1)^{t}\left[\Phi\left(\diamond_{d+2}^{k-1}\right)-\Phi\left(\diamond_{d+2}^{k}\right)\right] \\
& -t x(x-1)^{t-1}\left[\Phi\left(L_{v_{k+1}}\left(\diamond_{d+2}^{k-1}\right)\right)-\Phi\left(L_{v_{k+2}}\left(\diamond_{d+2}^{k}\right)\right)\right] \\
= & x^{2}(x-1)^{t-1}(x-2) u(x)
\end{aligned}
$$

where $u(x)=\left(x^{2}-(t+5) x+4\right) \Phi\left(D_{d-2 k}\right)+t(x-2) \Phi\left(D_{d-2 k-1}\right)$. When $k-1<d-k-1$, by Lemma 11(ii), we get

$$
\begin{aligned}
u(x)= & (x-2)\left(x^{2}-(t+5) x+t+4\right) \Phi\left(D_{d-2 k-1}\right) \\
& -\left(x^{2}-(t+5) x+4\right) \Phi\left(D_{d-2 k-2}\right) .
\end{aligned}
$$

Denote by $e(t)$ the largest root of $x^{2}-(t+5) x+4=0$. Then

$$
\begin{aligned}
& x^{2}-(t+5) x+4 \leq 0, \quad \text { if } x \in(t+4, e(t)], \\
& x^{2}-(t+5) x+t+4 \\
& \quad>x^{2}-(t+5) x+4>0, \quad \text { if } x>e(t) .
\end{aligned}
$$

Hence, by Lemma 12(i), (37) is greater than 0 when $x \geq$ $\mu_{1}\left(\diamond_{n}^{k}\right)$. So $\mu_{1}\left(\diamond_{n}^{k-1}\right)<\mu_{1}\left(\diamond_{n}^{k}\right)$ follows from Lemma 8 .

When $k-1=d-k-1$, (37) becomes

$$
\begin{aligned}
\Phi & \left(\diamond_{n}^{k-1}\right)-\Phi\left(\diamond_{n}^{k}\right) \\
& =x^{2}(x-1)^{t-1}(x-2)\left[\left(x^{2}-(t+5) x+4\right)\right] .
\end{aligned}
$$

If $t=1$, let $\diamond_{7}^{2}$ and $\diamond_{7}^{1}$ be two graphs with $d=4$. Through Maple 15, the largest root of $x^{2}-6 x+4=0$ is $e(1)=5.2363$ (up to four decimal places), which is less than $\mu_{1}\left(\diamond_{7}^{2}\right)=5.3145$ and $\mu_{1}\left(\diamond_{7}^{1}\right)=5.3008$. From Lemma 5, $\mu_{1}\left(\diamond_{7}^{2}\right) \leq \mu_{1}\left(\diamond_{n}^{k}\right)$ and $\mu_{1}\left(\diamond_{7}^{1}\right) \leq \mu_{1}\left(\diamond_{n}^{k-1}\right)$ hold when $n \geq 7$. So, (40) is greater than 0 for $x \geq \mu_{1}\left(\diamond_{n}^{k}\right)$. By Lemma $8, \mu_{1}\left(\diamond_{n}^{k-1}\right)<\mu_{1}\left(\diamond_{n}^{k}\right)$ holds.

If $t \geq 2$, by Lemmas 11(ii) and 12(iii),

$$
\begin{aligned}
& t(x)-\left[\left(x^{2}-(t+5) x+4\right)\right] \\
& \quad \times\left[(x-2) \Phi^{2}\left(D_{m}\right)-(t+4) \Phi\left(D_{m}\right) \Phi\left(D_{m-1}\right)\right] \\
& =2 t(x-1) \Phi^{2}\left(D_{m}\right)-\left[\left(t^{2}+3 t+4\right) x-4\right] \\
& \quad \times \Phi\left(D_{m}\right) \Phi\left(D_{m-1}\right)+2(t+2)(x-1) \Phi^{2}\left(D_{m-1}\right) \\
& =\left[2 t x^{2}-\left(t^{2}+9 t+4\right) x+(4 t+4)\right] \Phi\left(D_{m}\right) \Phi\left(D_{m-1}\right) \\
& \quad-2 t(x-1) \Phi\left(D_{m}\right) \Phi\left(D_{m-2}\right) \\
& \quad+2(t+2)(x-1) \Phi^{2}\left(D_{m-1}\right) \\
& =\left[2 t x^{2}-\left(t^{2}+9 t+4\right) x+(4 t+4)\right] \Phi\left(D_{m}\right) \Phi\left(D_{m-1}\right) \\
& \quad+4(x-1) \Phi^{2}\left(D_{m-1}\right)+2 t(x-1),
\end{aligned}
$$

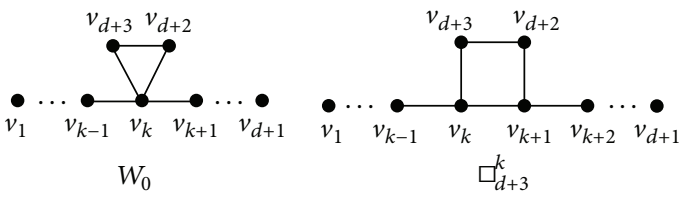

(a)

(b)

FIGURE 2

where

$$
\begin{aligned}
t(x)= & {\left[x^{3}-(t+7) x^{2}+(4 t+14) x-(2 t+8)\right] } \\
& \times \Phi^{2}\left(D_{m}\right)-\left[(t+4) x^{2}-(6 t+16) x+(4 t+12)\right] \\
& \times \Phi\left(D_{m}\right) \Phi\left(D_{m-1}\right)+2(t+2)(x-1) \Phi^{2}\left(D_{m-1}\right)
\end{aligned}
$$

(one may refer to (52) in Lemma 24).

By Lemma 12(ii), $(x-2) \Phi^{2}\left(D_{m}\right)-(t+4) \Phi\left(D_{m}\right) \Phi\left(D_{m-1}\right)>$ 0 when $x \geq t+4$. And by derivative, $2 t x^{2}-\left(t^{2}+9 t+4\right) x+$ $(4 t+4)>0$ when $x \geq t+4$.

Thus, (41) is greater than 0 for $x \geq e(t)>t+4$. From Lemma $8, \mu_{1}\left(\diamond_{n}^{k}\right)<e(t)$ holds. Put $x=\mu_{1}\left(\diamond_{n}^{k}\right)$ into (40), whose right side is less than 0 . So $\mu_{1}\left(\diamond_{n}^{k}\right)<\mu_{1}\left(\diamond_{n}^{k-1}\right)$. We complete the proof.

Form Lemma 20, the below corollary holds.

Corollary 21. When $1 \leq k \leq\lfloor d / 2\rfloor$,

(i) ifd is odd, then $\mu_{1}\left(\diamond_{n}^{k}\right) \leq \mu_{1}\left(\diamond_{n}^{\lfloor d / 2\rfloor}\right)$; the equality holds if and only if $\diamond_{n}^{k} \cong \diamond_{n}^{\lfloor d / 2\rfloor}$;

(ii) if $d$ is even, then

(a) when $n-d-2=0,1, \mu_{1}\left(\diamond_{n}^{k}\right) \leq \mu_{1}\left(\diamond_{n}^{\lfloor d / 2\rfloor}\right)$; the equality holds if and only if $\diamond_{n}^{k} \cong \diamond_{n}^{\lfloor d / 2\rfloor}$;

(b) when $n-d-2 \geq 2, \mu_{1}\left(\diamond_{n}^{k}\right) \leq \mu_{1}\left(\diamond_{n}^{\lfloor d / 2\rfloor-1}\right)$; the equality holds if and only if $\diamond_{n}^{k} \cong \diamond_{n}^{\lfloor d / 2\rfloor-1}$.

Let $W_{0}$ be the unicyclic graph of order $d+3$ shown in Figure 2. Let $W_{0}\left(p_{2}, \ldots, p_{d}, p_{d+2}, p_{d+3}\right)$ be a graph of order $n$ obtained from $W_{0}$ by attaching $p_{i}$ pendant vertices to each $v_{i} \in V\left(H_{0}\right) \backslash\left\{v_{1}, v_{d+1}\right\}$, respectively. When $k=2, p_{d+2}$ and $p_{d+3}=0$. Denote that

$$
\begin{aligned}
\widetilde{\mathscr{W}}_{n}^{d}= & \left\{W_{0}\left(p_{2}, \ldots, p_{d}, p_{d+2}, p_{d+3}\right):\right. \\
& \left.\sum_{i=2}^{d} p_{i}+p_{d+2}+p_{d+3}=n-d-3\right\},
\end{aligned}
$$

$$
\begin{aligned}
\overline{\mathscr{W}}_{n}^{d}=\{ & W_{0}\left(0, \ldots, 0, p_{i}, 0, \ldots, 0\right) \\
= & \left.W_{0}\left(p_{i}\right): p_{i}=n-d-3\right\} .
\end{aligned}
$$


Lemma 22. Let $G \in \widetilde{\mathscr{W}}_{n}^{d}$. Then there is a graph $G^{*} \in \overline{\mathscr{W}}_{n}^{d}$ such that $\mu_{1}\left(G^{*}\right) \geq \mu_{1}(G)$.

Proof. The proof is similar to that of Lemma 14.

Lemma 23. For any $G \in \overline{\mathscr{W}}_{n}^{d}, \mu_{1}(G) \leq \mu_{1}\left(W_{0}\left(p_{k}\right)\right)$, where $1 \leq$ $k-1 \leq d-k+1$; the equality holds if and only if $G \cong W_{0}\left(p_{k+1}\right)$.

Proof. Suppose that $n-d-3=t$. If $t=0$, the result is obvious.

If $t \geq 1$, by Lemma 7 , we have

$$
\begin{aligned}
& \mu_{1}(G) \\
& <\max \left\{\max \left\{d_{i}+m_{i} \mid v_{i} \in V(G)\right\} \mid G \in \overline{\mathscr{W}}_{n}^{d}, i \neq k\right\} \\
& =t+2+\frac{2+4+t}{t+2}<t+5=\Delta\left(W_{0}\left(p_{k}\right)\right)+1 \\
& <\mu_{1}\left(W_{0}\left(p_{k}\right)\right) .
\end{aligned}
$$

Hence, the lemma holds.

\section{Main Results and Their Proofs}

In this section, we first show that $\mu_{1}\left(\nabla_{n}^{\lfloor d / 2\rfloor+1}\right)<\mu_{1}\left(\Delta_{n}^{\lceil d / 2\rceil}\right)<$ $\mu_{1}\left(\diamond_{n}^{\lfloor d / 2\rfloor}\right)$.

Lemma 24. $\mu_{1}\left(\Delta_{n}^{\lceil d / 2\rceil}\right)<\mu_{1}\left(\diamond_{n}^{\lfloor d / 2\rfloor}\right)$.

Proof. Suppose that $n-d-2=t$; by Lemma 7, $t+4<$ $\mu_{1}\left(\Delta_{n}^{\lceil d / 2\rceil}\right), \mu_{1}\left(\diamond_{n}^{\lfloor d / 2\rfloor}\right)<t+5$ holds. We distinguish the following two cases.

Case 1. $d=2 m+1(m \geq 1)$.

Let $\Delta_{n}^{m+1}$ and $\diamond_{n}^{m}$ be two graphs on the left of Figure 3. If $t=0$, denote $\Delta_{n}^{m+1}$ and $\diamond_{n}^{m}$ by $G_{1}$ and $G_{2}$, respectively. Let $H_{1}=G_{1}-v_{m+3}-\cdots-v_{d+1}$ and $H_{2}=G_{2}-v_{m+3}-\cdots-v_{d+1}$. By Lemma 9,

$$
\begin{aligned}
\Phi\left(G_{1}\right)= & {\left[\Phi\left(P_{m}\right)-\Phi\left(L_{v_{m+2}}\left(P_{m}\right)\right)\right] \Phi\left(H_{1}\right) } \\
& -\Phi\left(P_{m}\right) \Phi\left(L_{v_{m+2}}\left(H_{1}\right)\right), \\
\Phi\left(G_{2}\right)= & {\left[\Phi\left(P_{m}\right)-\Phi\left(L_{v_{m+2}}\left(P_{m}\right)\right)\right] \Phi\left(H_{2}\right) } \\
& -\Phi\left(P_{m}\right) \Phi\left(L_{v_{m+2}}\left(H_{2}\right)\right),
\end{aligned}
$$

where

$$
\begin{aligned}
& \Phi\left(H_{1}\right)=x(x-3)\left[(x-3) \Phi\left(D_{m}\right)-2 \Phi\left(D_{m-1}\right)\right] \\
& \Phi\left(L_{v_{m+2}}\left(H_{1}\right)\right) \\
& \quad=(x-1)(x-3) \Phi\left(D_{m}\right)-(2 x-3) \Phi\left(D_{m-1}\right)
\end{aligned}
$$

$$
\begin{aligned}
& \Phi\left(H_{2}\right) \\
& =x(x-2) \\
& \quad \times\left[(x-2)(x-4) \Phi\left(D_{m-1}\right)-2(x-3) \Phi\left(D_{m-2}\right)\right], \\
& \Phi\left(L_{v_{m+2}}\left(H_{2}\right)\right) \\
& =(x-2)\left[\left(x^{2}-4 x+2\right) \Phi\left(D_{m-1}\right)-2(x-1) \Phi\left(D_{m-2}\right)\right] .
\end{aligned}
$$

Note that $\Phi\left(P_{m}\right)=x \Phi\left(D_{m-1}\right)$ and $\Phi\left(L_{v_{m+2}}\left(P_{m}\right)\right)=\Phi\left(D_{m-1}\right)+$ $\Phi\left(D_{m-2}\right)$.

Combining the equations above with Lemma 10, we get

$$
\begin{aligned}
& \Phi\left(\Delta_{n}^{m+1}\right) \\
& =(x-1)^{t-1}\left[(x-1) \Phi\left(G_{1}\right)-t x \Phi\left(L_{v_{m+2}}\left(G_{1}\right)\right)\right] \\
& =x(x-1)^{t-1}\left\{(x-1)(x-3)(x-3-t) \Phi^{2}\left(D_{m}\right)\right. \\
& \quad-\left[(t+4) x^{2}-(6 t+16) x+(6 t+12)\right] \\
& \quad \times \Phi\left(D_{m}\right) \Phi\left(D_{m-1}\right) \\
& \left.\quad+[(2 t+3) x-3(t+1)] \Phi^{2}\left(D_{m-1}\right)\right\} \\
& \Phi\left(\diamond_{n}^{m}\right) \quad(x-1)^{t-1}\left[(x-1) \Phi\left(G_{2}\right)-t x \Phi\left(L_{v_{m+2}}\left(G_{2}\right)\right)\right] \\
& =x(x-2)(x-1)^{t-1} \\
& \quad \times\left\{(x-1)\left[x^{3}-(t+8) x^{2}+(4 t+18) x-(2 t+10)\right]\right. \\
& \quad \times \Phi^{2}\left(D_{m-1}\right) \\
& \quad-\left[3 x^{3}-(3 t+19) x^{2}+8(t+4) x-4(t+4)\right] \\
& \quad \times \Phi\left(D_{m-1}\right) \Phi\left(D_{m-2}\right)+2(x-1)(x-3-t) \\
& \left.\quad \times \Phi^{2}\left(D_{m-1}\right)\right\} .
\end{aligned}
$$

Hence, by Lemmas 11(ii) and 12(i), when $\mu_{1}\left(\diamond_{n}^{m+1}\right) \leq x<t+5$,

$$
\Phi\left(\Delta_{n}^{m+1}\right)-\Phi\left(\diamond_{n}^{m}\right)
$$

$$
\begin{gathered}
=x(x-1)^{t}\left[d(x) \Phi^{2}\left(D_{m-1}\right)+e(x) \Phi\left(D_{m-1}\right) \Phi\left(D_{m-2}\right)\right. \\
\left.-r(x) \Phi^{2}\left(D_{m-2}\right)\right] \\
>x(x-1)^{t}[d(x)+e(x)-r(x)] \Phi^{2}\left(D_{m-2}\right) \geq 0,
\end{gathered}
$$


where

$$
\begin{gathered}
d(x)=-(x-1)(x-t-5)>0, \\
e(x)=\left(x^{2}-2 x+2\right)(x-t-4)>0, \\
r(x)=(x-1)(x-t-3)>0, \\
d(x)+e(x)-r(x)=(x-2)^{2}(x-t-4)>0 .
\end{gathered}
$$

So $\mu_{1}\left(\Delta_{n}^{m+1}\right)<\mu_{1}\left(\diamond_{n}^{m+1}\right)$ holds by Lemma 8 .

Case 2. $d=2 m+2(m \geq 1)$.

Let $\Delta_{n}^{m+1}$ and $\diamond_{n}^{m+1}$ be two graphs on the right of Figure 3. If $t=0$, denote $\Delta_{n}^{m+1}$ and $\diamond_{n}^{m+1}$ by $G_{1}^{*}$ and $G_{2}^{*}$, respectively. By similar computations as Case 1, we have

$$
\begin{aligned}
& \Phi\left(\Delta_{n}^{m+1}\right) \\
& =(x-1)^{t-1}\left[(x-1) \Phi\left(G_{1}^{*}\right)-t x \Phi\left(L_{v_{m+2}}\left(G_{1}^{*}\right)\right)\right] \\
& =x(x-1)^{t-1}\left\{(x-1)^{2}(x-3)(x-4-t) \Phi^{2}\left(D_{m}\right)\right. \\
& -(x-1)\left[3 x^{2}-(3 t+16) x+(6 t+18)\right] \\
& \times \Phi\left(D_{m}\right) \Phi\left(D_{m-1}\right) \\
& \left.+\left[2 x^{2}-2(t+4) x+3(t+2)\right] \Phi^{2}\left(D_{m-1}\right)\right\},
\end{aligned}
$$

$$
\begin{aligned}
& \Phi\left(\diamond_{n}^{m+1}\right) \\
&=(x-1)^{t-1}\left[(x-1) \Phi\left(G_{2}^{*}\right)-t x \Phi\left(L_{v_{m+3}}\left(G_{2}^{*}\right)\right)\right] \\
&=x(x-2)(x-1)^{t-1} \\
& \times\left\{\left[x^{3}-(t+7) x^{2}+(4 t+14) x-(2 t+8)\right] \Phi^{2}\left(D_{m}\right)\right. \\
&-\left[(t+4) x^{2}-(6 t+16) x+(4 t+12)\right] \\
& \times \Phi\left(D_{m}\right) \Phi\left(D_{m-1}\right) \\
&\left.+2(t+2)(x-1) \Phi^{2}\left(D_{m-1}\right)\right\} .
\end{aligned}
$$

Hence, by Lemmas 11(ii), 12(i) and 12(iii), when $x \geq t+4$,

$$
\begin{aligned}
\Phi\left(\Delta_{n}^{m+1}\right)-\Phi\left(\diamond_{n}^{m+1}\right) \\
=x(x-1)^{t} \\
\times\left\{-\left[(t+1) x^{2}-(3 t+5) x+(t+4)\right] \Phi^{2}\left(D_{m}\right)\right. \\
+\left[(t+1) x^{3}-(5 t+5) x^{2}+(7 t+10) x-(2 t+6)\right] \\
\times \\
\quad \Phi\left(D_{m}\right) \Phi\left(D_{m-1}\right) \\
\left.\quad-\left[(2 t+2) x^{2}-(4 t+4) x+(t+2)\right] \Phi^{2}\left(D_{m-1}\right)\right\}
\end{aligned}
$$

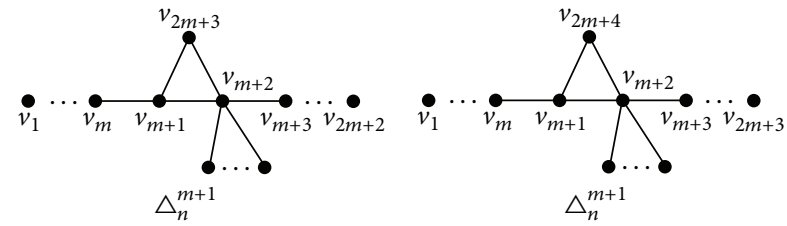

(a)

(b)

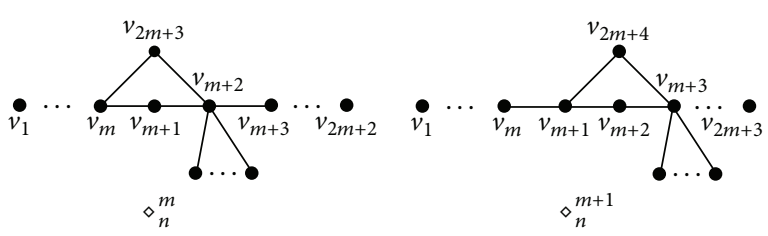

(c)

(d)

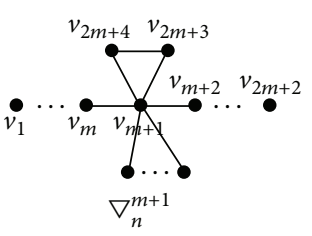

(e)

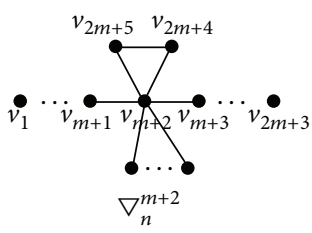

(f)
Figure 3

$$
\begin{aligned}
= & x(x-1)^{t} \\
& \times\left\{\left[p(x) \Phi\left(D_{m-1}\right)-q(x) \Phi\left(D_{m-2}\right)\right] \Phi\left(D_{m-1}\right)-w(x)\right\}, \\
\geq & x(x-1)^{t} \Phi\left(D_{m-1}\right) \\
& \times\left[(p(x)-w(x)) \Phi\left(D_{m-1}\right)-q(x) \Phi\left(D_{m-2}\right)\right]>0,
\end{aligned}
$$

where

$$
\begin{gathered}
p(x)=2 x^{3}-(t+9) x^{2}+(t+9) x-2>0, \\
q(x)=2(x-1)^{2}>0, \\
w(x)=(t+1) x^{2}-(3 t+5) x+(t+4)>0, \\
p(x)-w(x)-q(x) \\
=2 x^{3}-(2 t+12) x^{2}+(4 t+18) x-(t+8)>0 .
\end{gathered}
$$

So $\mu_{1}\left(\Delta_{n}^{m+1}\right)<\mu_{1}\left(\diamond_{n}^{m+1}\right)$ follows from Lemma 8 .

Let $\nabla_{n}^{k}$ be a graph of order $n$ obtained from a triangle by attaching $n-d-3$ pendant edges, a path of length $k-1$ and a path of length $d-k+1$ at one vertex of the triangle, where $1 \leq k-1 \leq d-k+1$.

Lemma 25. $\mu_{1}\left(\nabla_{n}^{\lfloor d / 2\rfloor+1}\right)<\mu_{1}\left(\Delta_{n}^{\lceil d / 2\rceil}\right)$.

Proof. Suppose that $n-d-3=t$; by Lemma 7(i), $\mu_{1}\left(\nabla_{n}^{\lfloor d / 2\rfloor+1}\right), \mu_{1}\left(\Delta_{n}^{\lceil d / 2\rceil}\right)>t+5$ holds. We distinguish the following two cases.

Case 1.d $d=2 m+1(m \geq 1)$. 
Let $\nabla_{n}^{m+1}$ be a graph on the left of Figure 3. If $t=0$, denote $\nabla_{n}^{m+1}$ by $G_{3}$. Let $H_{3}=G_{3}-v_{1}-\cdots-v_{m}$. By Lemma 9,

$$
\begin{aligned}
\Phi\left(G_{3}\right)= & {\left[\Phi\left(P_{m}\right)-\Phi\left(L_{v_{m}}\left(P_{m}\right)\right)\right] \Phi\left(H_{3}\right) } \\
& -\Phi\left(P_{m}\right) \Phi\left(L_{v_{m+1}}\left(H_{3}\right)\right),
\end{aligned}
$$

where

$$
\begin{aligned}
\Phi\left(H_{3}\right)= & x(x-3) \\
& \times\left[(x-1)(x-4) \Phi\left(D_{m}\right)-(x-3) \Phi\left(D_{m-1}\right)\right] \\
\Phi\left(L_{v_{m+1}}\left(H_{3}\right)\right)= & (x-1)(x-3) \\
& \times\left[(x-1) \Phi\left(D_{m}\right)-\Phi\left(D_{m-1}\right)\right] .
\end{aligned}
$$

By Lemma 10, we get

$$
\begin{aligned}
& \Phi\left(\nabla_{n}^{m+1}\right) \\
& =(x-1)^{t-1}\left[(x-1) \Phi\left(G_{3}\right)-t x \Phi\left(L_{v_{m+1}}\left(G_{3}\right)\right)\right] \\
& =x(x-3)(x-1)^{t} \\
& \quad \times\left\{(x-1)(x-t-4) \Phi^{2}\left(D_{m}\right)-[(t+4) x-(2 t+6)]\right. \\
& \left.\quad \times \Phi\left(D_{m}\right) \Phi\left(D_{m-1}\right)+(t+2) \Phi^{2}\left(D_{m-1}\right)\right\} .
\end{aligned}
$$

By (47), we have

$$
\begin{aligned}
\Phi\left(\Delta_{n}^{m+1}\right)=x & (x-1)^{t} \\
\times & \left\{(x-1)(x-3)(x-t-4) \Phi^{2}\left(D_{m}\right)\right. \\
& -\left[(t+5) x^{2}-(6 t+22) x+(6 t+18)\right] \\
& \times \Phi\left(D_{m}\right) \Phi\left(D_{m-1}\right) \\
& \left.+[(2 t+5) x-3(t+2)] \Phi^{2}\left(D_{m-1}\right)\right\} .
\end{aligned}
$$

Hence,

$$
\begin{aligned}
& \Phi\left(\nabla_{n}^{m+1}\right)-\Phi\left(\Delta_{n}^{m+1}\right) \\
& =x^{2}(x-1)^{t} \Phi\left(D_{m-1}\right) \\
& \quad \times\left[(x-t-4) \Phi\left(D_{m}\right)-(t+3) \Phi\left(D_{m-1}\right)\right]
\end{aligned}
$$

When $m=1,(59)=x^{2}(x-1)^{t+1}(x-t-5)>0$ holds for $x>t+5$. So $\mu_{1}\left(\nabla_{n}^{m+1}\right)<\mu_{1}\left(\Delta_{n}^{m+1}\right)$ follows from Lemma 8 .

When $m \geq 2, \mu_{1}\left(\nabla_{n}^{m+1}\right)<\mu_{1}\left(\Delta_{n}^{m+1}\right)$ also holds (the proof will be given in Case 2 of the lemma).

Case 2. $d=2 m+2(m \geq 1)$.
Let $\nabla_{n}^{m+2}$ be a graph on the right of Figure 3. By similar computations as in Case 1, we have

$$
\begin{aligned}
\Phi\left(\nabla_{n}^{m+2}\right)=x & (x-3)(x-1)^{t} \\
\times & \left\{(x-1)^{2}(x-t-5) \Phi^{2}\left(D_{m}\right)\right. \\
& -2(x-1)(x-t-4) \Phi\left(D_{m}\right) \Phi\left(D_{m-1}\right) \\
& \left.+(x-t-3) \Phi^{2}\left(D_{m-1}\right)\right\} .
\end{aligned}
$$

By (51), we get

$$
\begin{aligned}
& \Phi\left(\Delta_{n}^{m+1}\right) \\
& \begin{aligned}
=x(x-1)^{t-1}\{ & (x-1)^{2}(x-3)(x-t-5) \Phi^{2}\left(D_{m}\right) \\
& -(x-1)\left[3 x^{2}-(3 t+19) x+(6 t+24)\right] \\
& \times \Phi\left(D_{m}\right) \Phi\left(D_{m-1}\right) \\
& \left.+\left[2 x^{2}-2(t+5) x+(3 t+9)\right] \Phi^{2}\left(D_{m-1}\right)\right\} .
\end{aligned}
\end{aligned}
$$

Hence,

$$
\begin{aligned}
\Phi\left(\nabla_{n}^{m+2}\right)-\Phi\left(\Delta_{n}^{m+1}\right) \\
=x^{2}(x-1)^{t} \Phi\left(D_{m-1}\right) \\
\quad \times\left[(x-t-4) \Phi\left(D_{m+1}\right)-(t+3) \Phi\left(D_{m}\right)\right] .
\end{aligned}
$$

Let $t_{k}(x)=(x-t-4) \Phi\left(D_{k}\right)-(t+3) \Phi\left(D_{k-1}\right)$ and the largest root of $t_{k}(x)=0$ is denoted by $\mu_{1}\left(t_{k}(x)\right)$, where $k \geq 0$.

We first show that $\mu_{1}\left(t_{k}(x)\right)$ is strictly increasing.

We use the induction on $k$. Clearly, $\mu_{1}\left(t_{0}(x)\right)=t+4<$ $\mu_{1}\left(t_{1}(x)\right)=t+5$ holds. Generally, assume that $\mu_{1}\left(t_{k-1}(x)\right)<$ $\mu_{1}\left(t_{k}(x)\right)$, then by Lemmas 11(ii) and 12(iii),

$$
\begin{gathered}
t_{k+1}(x)=(x-2) t_{k}(x)-t_{k-1}(x), \\
t_{k}^{2}(x)-t_{k+1}(x) t_{k-1}(x) \\
=-\left[(t+2) x^{2}-(t+2)(t+5) x-1\right]=-v(x) .
\end{gathered}
$$

Put $x=\mu_{1}\left(t_{k}(x)\right)$ into (63), whose right side is less than 0 . So $\mu_{1}\left(t_{k}(x)\right)<\mu_{1}\left(t_{k+1}(x)\right)$.

Furthermore, $\mu_{1}\left(t_{k}(x)\right)$ has the upper bound.

Denote that the largest root of $v(x)=0$ is $\mu_{1}(v(x))$. If there exists some $m$ such that $\mu_{1}\left(t_{m}(x)\right) \geq \mu_{1}(v(x))$, we substitute $k$ with $m$ and put $x=\mu_{1}\left(t_{m}(x)\right)$ into (64). So, the right side of it is less than and equal to 0 and the left side is greater than 0 , a contradiction.

Let $G_{3}^{\prime}=\nabla_{n}^{m+2}-v_{1}-\cdots-v_{m-1}-v_{m+5}-\cdots-v_{2 m+3}$ and $G_{1}^{\prime}=\Delta_{n}^{m+1}-v_{1}-\cdots-v_{m-1}-v_{m+5}-\cdots-v_{2 m+3}$. 
By (60) and (62),

$$
\begin{aligned}
& \Phi\left(G_{3}^{\prime}\right)=x(x-1)^{t}(x-3)\left(x^{2}-3 x+1\right) r(x), \\
\Phi & \left(G_{3}^{\prime}\right)-\Phi\left(G_{1}^{\prime}\right) \\
= & x^{2}(x-1)^{t}\left[x^{3}-(t+8) x^{2}+(3 t+16) x-(t+6)\right] \\
= & x^{2}(x-1)^{t}(r(x)+1),
\end{aligned}
$$

where $r(x)=x^{3}-(t+8) x^{2}+(3 t+16) x-(t+7)$.

By Lemma 7(i), $\mu_{1}\left(G_{3}^{\prime}\right)$ is the largest root of $r(x)=0$. If $\mu_{1}\left(G_{3}^{\prime}\right) \geq \mu_{1}\left(G_{1}^{\prime}\right)$, then $\left.\left[\Phi\left(G_{3}^{\prime}\right)-\Phi\left(G_{1}^{\prime}\right)\right]\right|_{x=\mu_{1}\left(G_{3}^{\prime}\right)} \leq 0$ and $r(x)+$ $\left.1\right|_{x=\mu_{1}\left(G_{3}^{\prime}\right)}>0$, a contradiction. So $\mu_{1}\left(G_{3}^{\prime}\right)<\mu_{1}\left(G_{1}^{\prime}\right)$.

By derivative, when $x>t+5$,

$$
\begin{aligned}
& x v(x)-(t+2) r(x) \\
& =3(t+2) x^{2}-\left(3 t^{2}+22 t+33\right) x+(t+2)(t+7)>0
\end{aligned}
$$

From Lemma $8, \mu_{1}(v(x))<\mu_{1}\left(G_{3}^{\prime}\right)$ holds. Furthermore, $\mu_{1}\left(G_{3}^{\prime}\right) \leq \mu_{1}\left(\nabla_{n}^{m+2}\right)$ and $\mu_{1}\left(G_{1}^{\prime}\right) \leq \mu_{1}\left(\Delta_{n}^{m+1}\right)$ hold by Lemma 5 . Hence, (62) is greater than 0 for $x \geq \mu_{1}\left(\Delta_{n}^{m+1}\right)$. From Lemma 8 , we get $\mu_{1}\left(\nabla_{n}^{m+2}\right)<\mu_{1}\left(\Delta_{n}^{m+1}\right)$ as desired.

Let $\square_{d+3}^{k}$ be the unicyclic graph of order $d+3$ shown in Figure 2.

Lemma 26. $\mu_{1}\left(\square_{d+3}^{\lceil d / 2\rceil}\right)<\mu_{1}\left(\Delta_{d+3}^{\lceil d / 2\rceil}\right)$.

Proof. Note that by Lemma $7(\mathrm{i}), \mu_{1}\left(\Delta_{d+3}^{\lceil d / 2\rceil}\right)>5$.

Case 1. $d=2 m+1(m \geq 1)$.

By Lemma 9, we have

$$
\begin{aligned}
\Phi\left(\square_{2 m+4}^{m+1}\right)= & {\left[\Phi\left(P_{m}\right)-\Phi\left(L_{v_{m}}\left(P_{m}\right)\right)\right] } \\
& \times \Phi\left(H_{4}\right)-\Phi\left(P_{m}\right) \Phi\left(L_{v_{m+1}}\left(H_{4}\right)\right) \\
= & x\left[(x-2)^{2}(x-4) \Phi^{2}\left(D_{m}\right)-4(x-2)(x-3)\right. \\
& \left.\times \Phi\left(D_{m}\right) \Phi\left(D_{m-1}\right)+(3 x-8) \Phi^{2}\left(D_{m-1}\right)\right],
\end{aligned}
$$

where $H_{4}=\square_{2 m+4}^{m+1}-v_{1}-\cdots-v_{m}$.

By (47), we get

$$
\begin{aligned}
& \Phi\left(\Delta_{2 m+4}^{m+1}\right) \\
& =x\left[(x-1)(x-3)(x-4) \Phi^{2}\left(D_{m}\right)-\left(5 x^{2}-22 x+18\right)\right. \\
& \left.\quad \times \Phi\left(D_{m}\right) \Phi\left(D_{m-1}\right)+(5 x-6) \Phi^{2}\left(D_{m-1}\right)\right] .
\end{aligned}
$$

Hence, by Lemmas 11(ii), 12(ii), and 12(iii), when $x \geq 5$,

$$
\begin{aligned}
& \Phi\left(\square_{2 m+4}^{m+1}\right)-\Phi\left(\Delta_{2 m+4}^{m+1}\right) \\
& =x\left[(x-4) \Phi^{2}\left(D_{m}\right)+\left(x^{2}-2 x-6\right) \Phi\left(D_{m}\right) \Phi\left(D_{m-1}\right)\right. \\
& \left.\quad-2(x+1) \Phi^{2}\left(D_{m-1}\right)\right]
\end{aligned}
$$

$$
\begin{aligned}
= & x \Phi\left(D_{m-1}\right)\left[2\left(x^{2}-4 x+1\right) \Phi\left(D_{m}\right)-(3 x-2) \Phi\left(D_{m-1}\right)\right] \\
& +x(x-4) \\
> & x \Phi^{2}\left(D_{m-1}\right)\left(4 x^{2}-19 x+6\right)+x(x-4)>0 .
\end{aligned}
$$

So $\mu_{1}\left(\square_{2 m+4}^{m+1}\right)<\mu_{1}\left(\Delta_{2 m+4}^{m+1}\right)$ follows from Lemma 8 .

Case 2. $d=2 m+2(m \geq 1)$. holds.

By a similar proof as of Case $1, \mu_{1}\left(\square_{2 m+5}^{m+1}\right)<\mu_{1}\left(\Delta_{2 m+5}^{m+1}\right)$

Next we give the proof of Theorem 1, which is the most important result.

Proof of Theorem 1. Let $G \in \mathscr{C}_{n}^{d}$ and $X=\left(x_{1}, x_{2}, \ldots, x_{n}\right)^{T}$ be a unit eigenvector of $\mu_{1}(G)$, where $x_{i}$ corresponds to the vertex $v_{i}(1 \leq i \leq n)$.

Choose $G \in \mathscr{C}_{n}^{d}$ such that the Laplacian spectral radius of $G$ is as large as possible. Then, by Lemma 6 , we can assume that $G \neq C_{n}$. Let $P_{d+1}=v_{1} v_{2}, \ldots, v_{d+1}$ be the induced path of length $d$ and let $C_{q}$ be the only cycle in $G$. Since $G \neq C_{n}$, we have $\min \left\{d\left(v_{1}\right), d\left(v_{d+1}\right)\right\}=1$, say $d\left(v_{1}\right)=1$. We first show some claims.

Claim 1. $V\left(C_{q}\right) \cap V\left(P_{d+1}\right) \neq \emptyset$.

Proof of Claim 1. Otherwise, since $G$ is connected, there exists an only path $P=v_{i} v_{k} v_{k+1}, \ldots, v_{l-1} v_{l}$ connecting $C_{q}$ and $P_{d+1}$, where $v_{i} \in V\left(C_{q}\right), v_{l} \in V\left(P_{d+1}\right)$, and $v_{k}, \ldots, v_{l-1} \in V(G) \backslash$ $\left(V\left(C_{q}\right) \cup V\left(P_{d+1}\right)\right)$.

For each $j$, let $T_{j}$ be a rooted tree (with $r_{j}$ as its root) attached at $v_{j}(k \leq j \leq l-1)$, where the order of $T_{j}$ is $n_{j}$. We assume that all trees, but $T_{i}$, are kept fixed, while $T_{i}$ (along with its root) can be changed. Suppose that $T_{i} \neq S_{n_{i}}$. Let $v$ be a vertex belonging to $T_{i}$ chosen so that $d(v)>2$ and that $d\left(v, r_{i}\right)$ (the distance between $v$ and $r_{i}$ ) is the largest. By Lemma 4 (applied in the reverse direction), the Laplacian spectral radius is increased when any hanging path at $v$ is replaced by a hanging star (namely, edges of a hanging path now become the hanging edges at $v$ ). If the same is repeated for other hanging paths at $v$, we get one star attached at $v$ (its central vertex is identified with $v$ ) whose size is equal to the sum of the lengths of the aforementioned paths. Let $w$ be a vertex in $T_{i}$, adjacent to $v$, and belonging to the (unique) path between $r_{i}$ and $v$. By Lemma 3, the Laplacian spectral radius is increased when all hanging edges at $v$ become the hanging edges at $w$. Note also that $d\left(w, r_{i}\right)=d\left(v, r_{i}\right)-1$. By repeating the same procedure (for any other vertex as $v$ ), we arrive at $\widetilde{G}_{1}$, where the rooted tree $T_{i}$ becomes a star $S_{n_{i}}$, so that $\mu_{1}\left(\widetilde{G}_{1}\right) \geq \mu_{1}(G)$.

By the same way to other rooted trees, we arrive at $\widetilde{G}_{2}$, where every rooted tree $T_{j} \cong S_{n_{j}}(k \leq j \leq l-1)$, and $\mu_{1}\left(\widetilde{G}_{2}\right) \geq$ $\mu_{1}\left(\widetilde{G}_{1}\right)$. From $\widetilde{G}_{2}$, applying Lemma 2 , we arrive at $\widetilde{G}_{3}$, where only a rooted tree $S_{n_{k}+\cdots+n_{l-1}}$ is attached at some vertex $v_{m} \in$ $\left\{v_{k}, \ldots, v_{l-1}\right\}$. So $\mu_{1}\left(\widetilde{G}_{3}\right) \geq \mu_{1}\left(\widetilde{G}_{2}\right)$.

From $\widetilde{G}_{3}$, by Lemma 3 , the Laplacian spectral radius increased when all vertices adjacent to $v_{l}$ become adjacent 
vertices to $v_{l-1}$. By repeating the same procedure (for any other vertex of $P-v_{i}$ as $\left.v_{l}\right)$, we arrive at $\widetilde{G}_{4}$, where $V\left(C_{q}\right) \cap V\left(P_{d+1}\right) \neq \emptyset$, and $\mu_{1}\left(\widetilde{G}_{4}\right) \geq \mu_{1}\left(\widetilde{G}_{3}\right)$.

Hence, we have $\mu_{1}\left(\widetilde{G}_{4}\right) \geq \mu_{1}(G)$, a contradiction.

By Claim 1, $V\left(C_{q}\right) \bigcap V\left(P_{d+1}\right) \neq \emptyset$. Denote that $C_{q}=v_{k} v_{k+1}, \ldots, v_{l-1} v_{l} v_{d+2} v_{d+3}, \ldots, v_{s} v_{k}(s \geq d+2)$, where $\left\{v_{k}, v_{k+1}, \ldots, v_{l-1}, v_{l}\right\}=V\left(C_{q}\right) \cap V\left(P_{d+1}\right)$ and $\left\{v_{d+2}, v_{d+3}, \ldots, v_{s}\right\}=V\left(C_{q}\right) \backslash V\left(P_{d+1}\right)$.

Claim 2. $d(v)=1$ for $v \in V(G) \backslash\left(V\left(C_{q}\right) \bigcup V\left(P_{d+1}\right)\right)$.

Proof of Claim 2. Consider other rooted trees attached at $V\left(C_{q}\right)$ and $V\left(P_{d+1}\right)$, respectively. By a similar proof as Claim 1 (the procedure until $\left.\widetilde{G}_{3}\right)$, we can get $\widetilde{H}_{i}$ and $\mu_{1}\left(\widetilde{H}_{i}\right) \geq$ $\mu_{1}(G)$, where only a rooted tree is attached at $v_{i}\left(v_{i} \in\right.$ $\left.\left\{V\left(C_{q}\right) \bigcup V\left(P_{d+1}\right)\right\} \backslash\left\{v_{1}, v_{d+1}\right\}\right)$, a contradiction.

Claim 3. $l=k+1$ and $s=d+2$.

Proof of Claim 3. Denote that $A=\{v|v \in V(G)|$ $\left(V\left(C_{q}\right) \bigcup V\left(P_{d+1}\right)\right\}=\left\{v_{s+1}, \ldots, v_{n}\right\}$ and $|A|=n-s=t \geq 0$.

Case 1. $l=k$. By Lemma 7,

$$
\begin{aligned}
\mu_{1}\left(\widetilde{H}_{i}\right) & \leq \max \left\{\max \left\{d_{v_{i}}+m_{v_{i}} \mid v_{i} \in V\left(\widetilde{H}_{i}\right)\right\} \mid i \neq k\right\} \\
& =t+2+\frac{2+4+t}{t+2} \leq t+5=\Delta\left(\widetilde{H}_{k}\right)+1 \\
& <\mu_{1}\left(\widetilde{H}_{k}\right)<\max \left\{d_{v_{i}}+m_{v_{i}} \mid v_{i} \in V\left(\widetilde{H}_{k}\right)\right\} \\
& =t+4+\frac{2+2+2+2+t}{t+4} \leq t+6 \leq n-d+2 \\
& =\Delta\left(\nabla_{n}^{k}\right)+1<\mu_{1}\left(\nabla_{n}^{k}\right)
\end{aligned}
$$

for $s \geq d+4\left(\left|C_{q}\right| \geq 4\right)$.

When $s=d+3\left(\left|C_{q}\right|=3\right), \widetilde{H}_{i} \cong W_{0}\left(p_{i}\right)$. By Lemma 23, $\mu_{1}\left(\widetilde{H}_{i}\right)<\mu_{1}\left(\nabla_{n}^{k}\right)$ for any $i \neq k$, where $\widetilde{H}_{k} \cong \nabla_{n}^{k}$.

Hence, by Lemma $4, \nabla_{n}^{\lfloor d / 2\rfloor+1}$ has the larger Laplacian spectral radius. Furthermore, from Lemmas 24 and 25, $\mu_{1}\left(\diamond_{n}^{\lfloor d / 2\rfloor}\right)>\mu_{1}\left(\nabla_{n}^{\lfloor d / 2\rfloor+1}\right)$ holds.

Case 2. $l=k+1$.

Subcase 2.1. $t \geq 1$. By Lemma 7,

$$
\begin{aligned}
& \mu_{1}\left(\widetilde{H}_{i}\right) \\
& \leq \max \left\{\max \left\{d_{v_{i}}+m_{v_{i}} \mid v_{i} \in V\left(\widetilde{H}_{i}\right)\right\}, i \neq k, k+1\right\} \\
& =t+2+\frac{2+3+t}{t+2} \leq t+4=\Delta\left(\widetilde{H}_{k}\right)+1\left(\Delta\left(\widetilde{H}_{k+1}\right)+1\right) \\
& <\mu_{1}\left(\widetilde{H}_{k}\right)\left(\mu_{1}\left(\widetilde{H}_{k+1}\right)\right)<\max \left\{d_{v_{i}}+m_{v_{i}} \mid v_{i} \in V\left(\widetilde{H}_{k}\right)\right\}
\end{aligned}
$$

$$
\begin{aligned}
& \leq t+3+\frac{2+3+t}{t+3}<t+5 \leq n-d+2=\Delta\left(\Delta_{n}^{k}\right)+1 \\
& <\mu_{1}\left(\Delta_{n}^{k}\right)
\end{aligned}
$$

for $s \geq d+3\left(\left|C_{q}\right| \geq 4\right)$.

When $s=d+2\left(\left|C_{q}\right|=3\right), \widetilde{H}_{i} \cong H_{0}\left(p_{i}\right)$, By Lemma 15, $\mu_{1}\left(\widetilde{H}_{i}\right)<\mu_{1}\left(\Delta_{n}^{k}\right)$ for any $i \neq k+1$, where $\widetilde{H}_{k+1} \cong \Delta_{n}^{k}$.

Subcase 2.2. $t=0$. By Lemma 7,

$$
\begin{aligned}
\mu_{1}\left(\widetilde{H}_{i}\right) & \leq \max \left\{\max \left\{d_{v_{i}}+m_{v_{i}} \mid v_{i} \in V\left(\widetilde{H}_{i}\right)\right\} i \neq 1, d+1\right\} \\
& =3+\frac{2+3+2}{3}<6<n-d+2=\Delta\left(\Delta_{n}^{k}\right)+1 \\
& <\mu_{1}\left(\Delta_{n}^{k}\right)
\end{aligned}
$$

for $s \geq d+4\left(\left|C_{q}\right| \geq 5\right)$.

When $s=d+3\left(\left|C_{q}\right|=4\right)$, by Lemma 13, $\mu_{1}\left(\square_{d+3}^{k}\right)<$ $\mu_{1}\left(\square_{d+3}^{[d / 2\rceil}\right)$. Furthermore, from Lemma 26, $\mu_{1}\left(\Delta_{d+3}^{\lceil d / 2\rceil}\right)>$ $\mu_{1}\left(\square_{d+3}^{\lceil d / 2\rceil}\right)$.

When $s=d+2\left(\left|C_{q}\right|=3\right), \widetilde{H}_{i} \cong H_{0}\left(p_{i}\right) \cong \Delta_{n}^{k}$.

Hence, in view of Subcases 2.1 and 2.2, by Corollary 17 and Lemma $24, \mu_{1}\left(\diamond_{n}^{\lfloor d / 2\rfloor}\right)>\mu_{1}\left(\Delta_{n}^{\lceil d / 2\rceil}\right)$ holds.

Case $3 . l \geq k+2$. By Lemma 7 ,

$$
\begin{aligned}
& \mu_{1}\left(\widetilde{H}_{i}\right) \\
& \quad \leq \max \left\{\max \left\{d_{v_{i}}+m_{v_{i}} \mid v_{i} \in V\left(\widetilde{H}_{i}\right)\right\} i \neq 1, d+1\right\} \\
& =t+3+\frac{2+2+2+t}{t+3} \leq t+5 \leq n-d+2=\Delta\left(\diamond_{n}^{k}\right)+1 \\
& \quad<\mu_{1}\left(\diamond_{n}^{k}\right)
\end{aligned}
$$

for $s \geq d+3\left(\left|C_{q}\right| \geq 5\right)$.

When $s=d+2\left(\left|C_{q}\right|=4\right), \widetilde{H}_{i} \cong U_{0}\left(p_{i}\right)$, by Lemma 19, $\mu_{1}\left(\widetilde{H}_{i}\right)<\mu_{1}\left(\diamond_{n}^{k}\right)$ for any $i \neq k+2$, where $\widetilde{H}_{k+2} \cong \diamond_{n}^{k}$.

Hence, in view of Cases 1, 2, and 3 and Corollary 21, if $d$ is odd, $\diamond_{n}^{\lfloor d / 2\rfloor}$ has the largest Laplacian spectral radius; if $d$ is even and $n-d-2=0,1, \diamond_{n}^{\lfloor d / 2\rfloor}$ has the largest Laplacian spectral radius; If $d$ is even and $n-d-2 \geq 2, \diamond_{n}^{\lfloor d / 2\rfloor-1}$ has the largest Laplacian spectral radius, a contradiction.

By Claims 1, 2, and 3, Theorem 1 follows immediately.

\section{Acknowledgments}

The author would like to express sincere gratitude to the referees for a very careful reading of the paper and for all their insightful comments and valuable suggestions, which led to improving this paper. The research of the author is supported by the National Natural Science Foundation of China (nos. 11371078 and 61303020) and the National Natural Science Foundation of Shanxi Province (no. 2012011019-2). 


\section{References}

[1] D. Cvetkovic, M. Doob, and H. Sachs, Spectra of Graphs, Academic Press, New York, NY, USA, 1976.

[2] J. X. Li, W. C. Shiu, and W. C. Han, "The Laplacian spectral radius of some graphs," Linear Algebra and Its Applications, vol. 431, no. 1-2, pp. 99-103, 2009.

[3] X. L. Zhang and H. P. Zhang, "The Laplacian spectral radius of some bipartite graphs," Linear Algebra and Its Applications, vol. 428, no. 7, pp. 1610-1619, 2008.

[4] S. G. Guo, "On the Laplacian spectral radius of unicyclic graphs with fixed diameter," Ars Combinatoria C, vol. 6, pp. 47-58, 2012.

[5] S. G. Guo, "The largest eigenvalues of Laplacian matrices of unicyclic graphs," Applied Mathematics A, vol. 16, no. 2, pp. 131135, 2001 (Chinese).

[6] S. S. He and S. C. Li, "On the signless Laplacian index of unicyclic graphs with fixed diameter," Linear Algebra and Its Applications, vol. 436, no. 1, pp. 252-261, 2012.

[7] M. H. Liu, X. Z. Tan, and B. L. Liu, "The (signless) Laplacian spectral radius of unicyclic and bicyclic graphs with $n$ vertices and $k$ pendant vertices," Czechoslovak Mathematical Journal, vol. 60 , no. 3, pp. 849-867, 2010.

[8] F. L. Hua, G. H. Yu, and A. Ilić, "The Laplacian spectral radius for unicyclic graphs with given independence number," Linear Algebra and Its Applications, vol. 433, no. 5, pp. 934-944, 2010.

[9] H. Q. Liu, M. Lu, and F. Tian, "On the spectral radius of unicyclic graphs with fixed diameter," Linear Algebra and Its Applications, vol. 420 , no. 2-3, pp. 449-457, 2007.

[10] J. M. Guo, "The Laplacian spectral radius of a graph under perturbation," Computers and Mathematics with Applications, vol. 54 , no. 5, pp. 709-720, 2007.

[11] J. M. Guo, "The effect on the Laplacian spectral radius of a graph by adding or grafting edges," Linear Algebra and Its Applications, vol. 413, no. 1, pp. 59-71, 2006.

[12] R. Grone and R. Merris, "The Laplacian spectrum of a graph," SIAM Journal on Discrete Mathematics, vol. 7, no. 2, pp. 221-229, 1994.

[13] R. Merris, “A note on Laplacian graph eigenvalues," Linear Algebra and Its Applications, vol. 285, no. 1-3, pp. 33-35, 1998.

[14] C. X. He, J. Y. Shao, and J. He, "On the Laplacian spectral radii of bicyclic graphs," Discrete Mathematics, vol. 308, no. 24, pp. 5981-5995, 2008.

[15] J. M. Guo, "A conjecture on the algebraic connectivity of connected graphs with fixed girth," Discrete Mathematics, vol. 308, no. 23, pp. 5702-5711, 2008. 


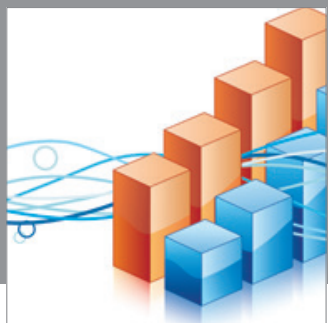

Advances in

Operations Research

mansans

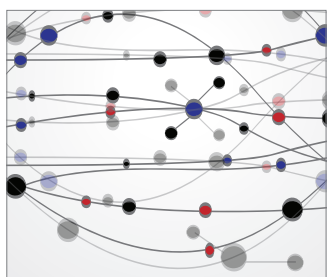

The Scientific World Journal
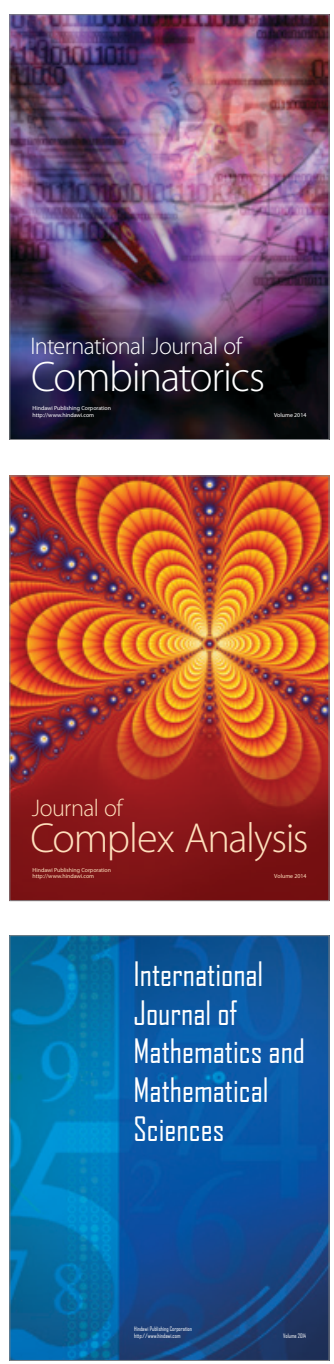
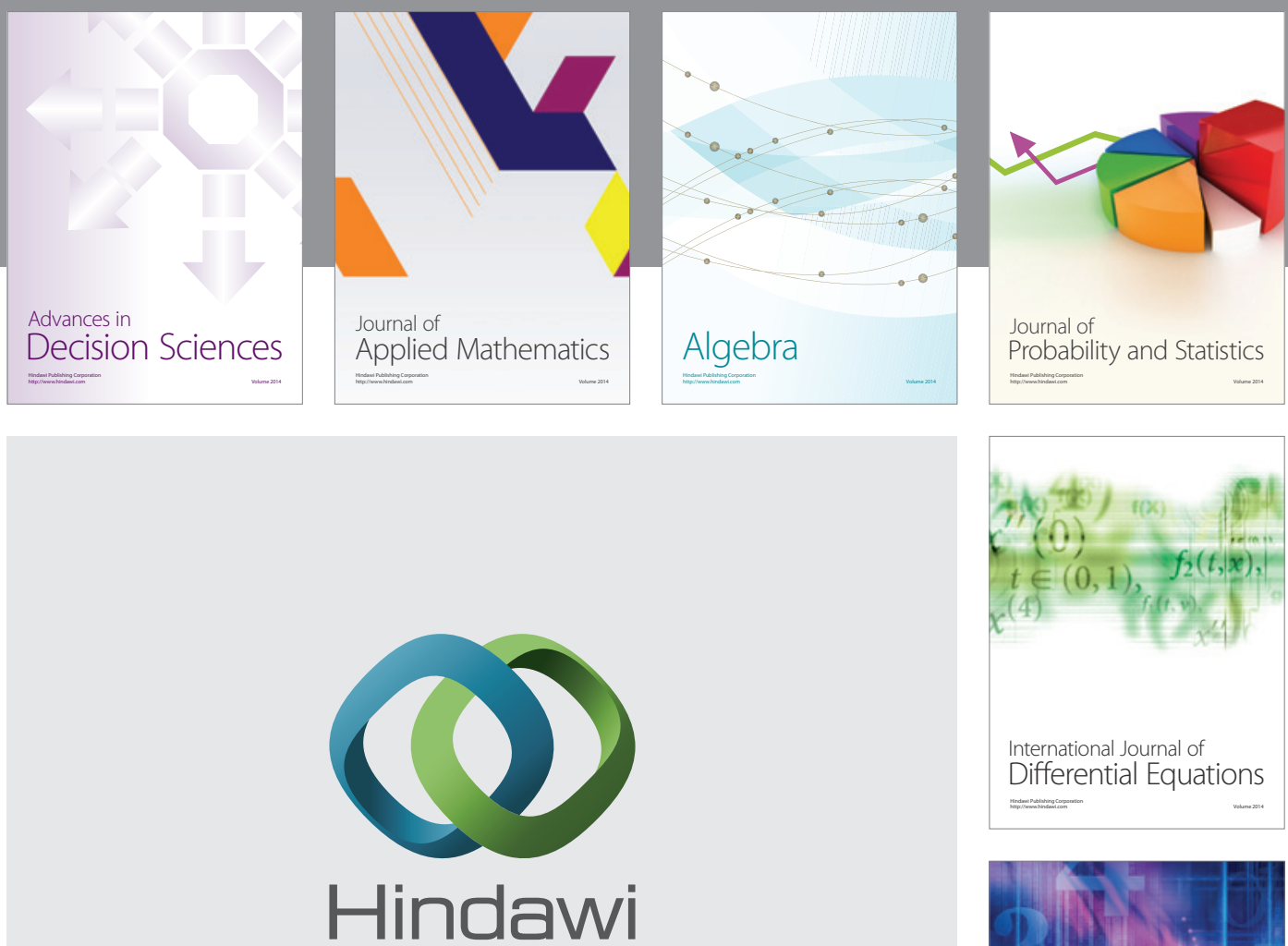

Submit your manuscripts at http://www.hindawi.com
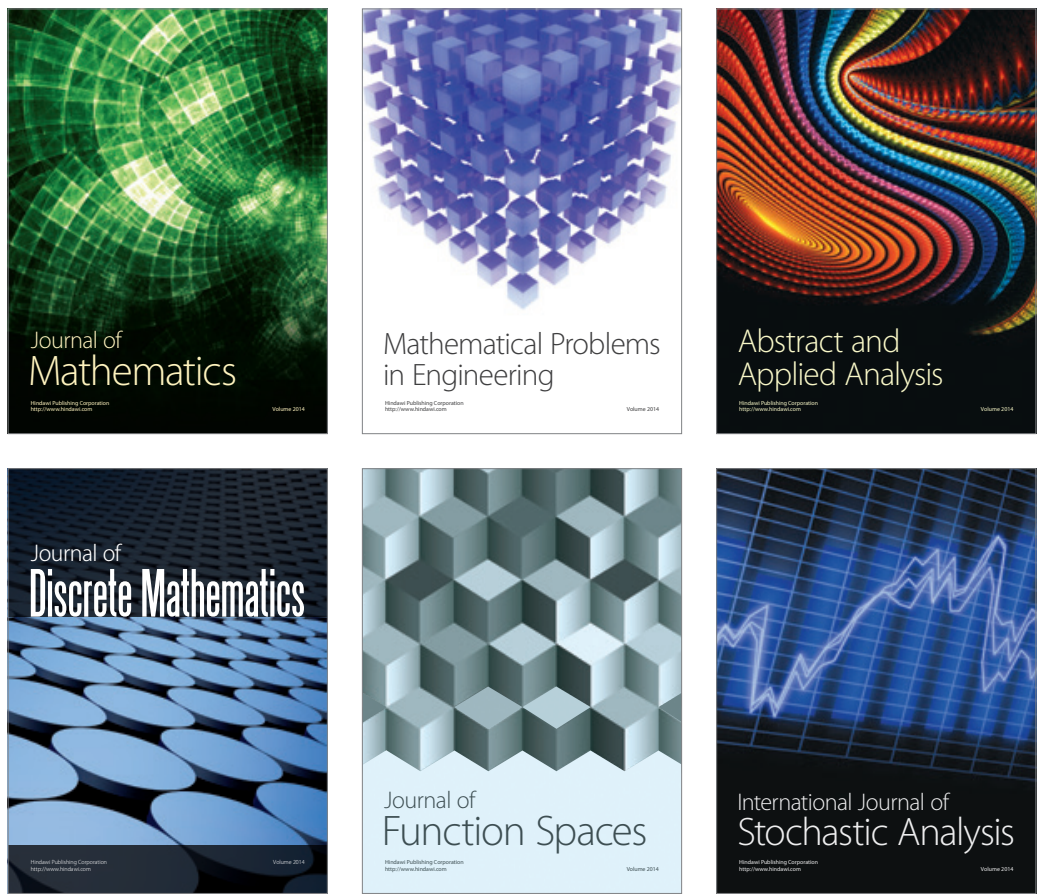

Journal of

Function Spaces

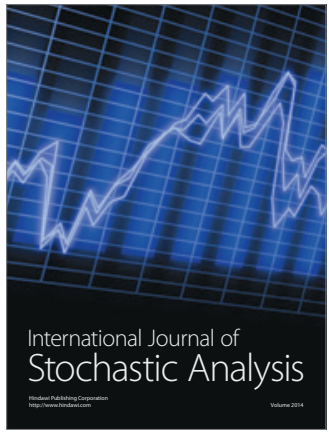

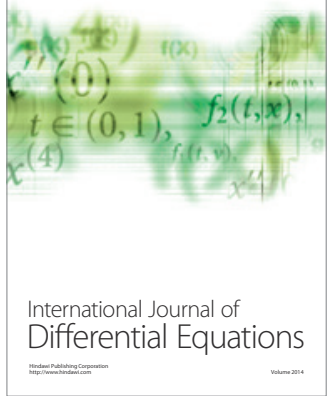
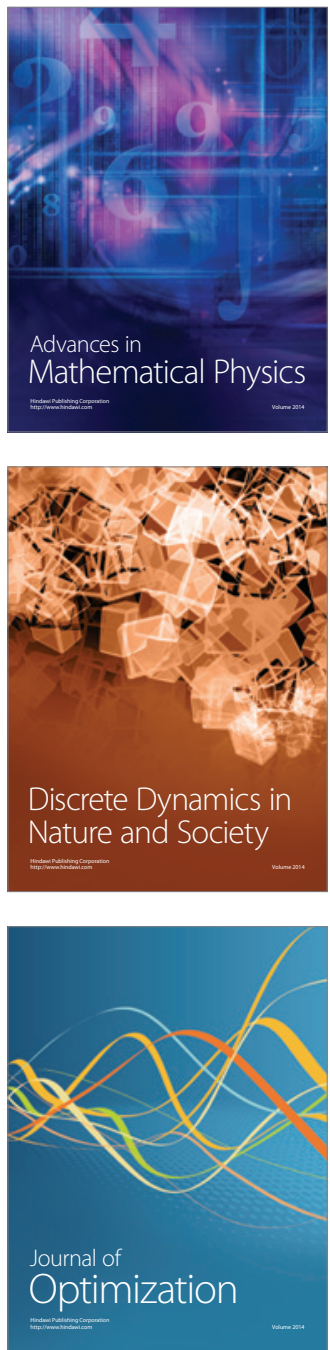